\title{
Geometric Aspects of Quantum Spin States
}

\author{
Michael Aizenman ${ }^{\star}$, Bruno Nachtergaele \\ Department of Physics, Princeton University, Jadwin Hall, P.O. Box 708, Princeton NJ 08544- \\ 0708, USA
}

Received: 18 June 1993/in revised form: 1 October 1993

\begin{abstract}
A number of interesting features of the ground states of quantum spin chains are analyzed with the help of a functional integral representation of the system's equilibrium states. Methods of general applicability are introduced in the context of the $\mathrm{SU}(2 S+1)$-invariant quantum spin- $S$ chains with the interaction $-P^{(0)}$, where $P^{(0)}$ is the projection onto the singlet state of a pair of nearest neighbor spins. The phenomena discussed here include: the absence of Néel order, the possibility of dimerization, conditions for the existence of a spectral gap, and a dichotomy analogous to one found by Affleck and Lieb, stating that the systems exhibit either slow decay of correlations or translation symmetry breaking. Our representation elucidates the relation, evidence for which was found earlier, of the $-P^{(0)}$ spin- $S$ systems with the Potts and the Fortuin-Kasteleyn random-cluster models in one more dimension. The method reveals the geometric aspects of the listed phenomena, and gives a precise sense to a picture of the ground state in which the spins are grouped into random clusters of zero total spin. E.g., within such structure the dichotomy is implied by a topological argument, and the alternatives correspond to whether, or not, the clusters are of finite mean length.
\end{abstract}

\section{Table of Contents}

1. Introduction . . . . . . . . . . . . . . . . . . . . . . . . . . 18

2. Quasi-State Decomposition for Equilibrium States of Quantum Spin Systems 21

Work supported in part by NSF Grant PHY-9214654.

* Also in the Mathematics Department.

E-mail: aizenman@math.princeton.edu bxn@math.princeton.edu

(C) 1993 Copyrights rest with the authors. Faithful reproduction of the article for non-commercial purpose is permitted. 
2.1. The Functional Integral Representation for the Spin- $S$ Model with Interaction $-P^{(0)}$

2.2. Poisson Process Representation $e^{-\beta H}$. . . . . . . . . . . . . . 24

2.3. The Q-S-Decomposition . . . . . . . . . . . . . . . . . . . 25

2.4. The Spin-1/2 Heisenberg Ferro- and Antiferromagnet . . . . . . . 25 2.4.a. The Ferromagnet $\left(H^{F}\right)$. . . . . . . . . . . . . . . . . 26

2.4.b. The Antiferromagnet $\left(H^{A F}\right)$. . . . . . . . . . . . . . . . . . 27

2.5. Structure of the Quasi-States . . . . . . . . . . . . . . . . . 29

2.6. The $S U(2 S+1)$-Invariant Spin- $S$ Models with the Interaction $-P^{(0)} \quad .30$

3. Equivalence with the 2-Dimensional $(2 S+1)^{2}$-State Potts-Models . . . . 31

4. Finite Systems and the Thermodynamic Limit . . . . . . . . . . . . 37

5. Absence of Néel Order . . . . . . . . . . . . . . . . . . . . . . 40

6. Dimerization versus Power Law Decay: A Dichotomy . . . . . . . . . 41

6.1. The Dichotomy . . . . . . . . . . . . . . . . . . . . . . . 42

6.2. The Dimerization Order Parameter . . . . . . . . . . . . . . . 44

7. Decay of Correlations in the Spin $S$ Model with Interaction $-P^{(0)}$. . . . 46

Appendix I: Quasi-State Decomposition for Quantum States . . . . . . . 56

Appendix II: FKG Structure and the Rising Tide Lemma . . . . . . . . . 59

References . . . . . . . . . . . . . . . . . . . . . . 61

\section{Introduction}

There is a geometric aspect to the structure of the spin-spin correlations found at low temperatures in a number of quantum spin systems. Our purpose is to introduce some generally applicable tools for the analysis of such phenomena. That is done in the context of the $\mathrm{SU}(2 S+1)$ invariant models introduced by Affleck [55] and also studied by Batchelor and Barber, and Klümper [6, 10, 11, 38, 39], which include the spin 1/2 Heisenberg antiferromagnet as a special case.

The systems considered here are one dimensional chains of spin $S$ variables, with the Hamiltonian

$$
H=-(2 S+1) \sum_{x} J_{x} P_{x, x+1}^{(0)}
$$

where $P_{x, y}^{(0)}$ is the orthogonal projection onto the singlet state of two quantum spins of magnitude $S$, and $J_{x}>0$. The models with translation invariant (all $J_{x}=J$ ) or staggered coupling constants (possibly with two different values for $x$ even and odd) are of special interest and some of our results are specific for these cases. We will refer to the Hamiltonians (1.1) as the spin $S$ models with interaction $-P^{(0)}$. The explicit form of the interaction in the basis of eigenstates of $S^{3}$ is:

$$
(2 S+1) P_{x, y}^{(0)}=\sum_{\alpha, \beta=-S}^{S}(-1)^{\alpha-\beta}|\beta,-\beta\rangle\langle\alpha,-\alpha| .
$$

The interaction can of course also be expressed as a polynomial in the Heisenberg interaction $\mathbf{S}_{x} \cdot \mathbf{S}_{x+1}$. For $S=1 / 2$ and $S=1$ one obtains

$$
P_{x, y}^{(0)}=\left\{\begin{array}{ll}
\frac{1}{4}-\mathbf{S}_{x} \cdot \mathbf{S}_{x+1} & \text { for } S=1 / 2 \\
\frac{1}{3}\left(\mathbf{S}_{x} \cdot \mathbf{S}_{x+1}\right)^{2}-\frac{1}{3} & \text { for } S=1
\end{array} .\right.
$$

The analogous expressions for general $S$ can be found, e.g., in [11]. 
The phenomena which we shall address are:

1) The nature of the order parameters which characterize the possible occurrence of symmetry breaking in the ground state.

i. Néel order. In higher dimensions such models may exhibit Néel order in the ground state. For the standard Heisenberg antiferromagnet this has been proved for dimensions $d \geqq 3$ and also for $d=2$ if $S \geqq 1[16,35]$. The representation introduced here permits to rule out that possibility for the translation invariant models with interaction (1.1) in one dimension, on the basis of known results in percolation theory (in two dimensions). (In one dimension, the representation relates Néel order to a transient behavior in a system of random loops which form the boundaries of the connected clusters of a random cluster model.)

ii. Dimerization. The one dimensional models may, nevertheless, exhibit a twofold translation symmetry breaking, caused by dimerization. While the interaction favors the pairing of neighboring spins into singlet states, not all neighboring spins can be paired simultaneously. There are, of course, states - corresponding to different dimerizations of the lattice, in which half (or, on the lattice $\mathbb{Z}^{d}$, a fraction $1 /(2 d)$ ) of the interaction terms are minimized. While these are not true ground states, it turns out that in one dimension for $S$ large enough $(S \geqq 1)$ this structure is present in the ground state, which decomposes into a superposition of two partially dimerized states. Spins on even sites have stronger correlations with their neighbors to the right in one of the states, and to the left in the other.

The classical dimerization picture is too naive in two aspects: 1) the model's correlation functions extend beyond nearest neighbors, and 2) the spins correlate in larger clusters than pairs. A virtue of the method employed here is that it permits to describe this phenomenon (and the picture of the correlated clusters) in explicit and precise terms. In particular, we find the following behavior: in the state where the spins on the even sites are more correlated with their neighbors to the right one finds that with probability 1 some spins on the left half-infinite chain $(-\infty, x]$ form a singlet with some spins on the right half-infinite chain $[x+1,+\infty)$, for each $x$ even. In the same state this probability is $<1$ for $x$ odd.

2) The "dimerization versus power law decay" dichotomy. The dimerization does not always occur. However, we show that there is a dichotomy: the ground state either dimerizes, or exhibits slow decay of correlations (with $\sum_{x}\left|x\left\langle S_{0}^{3} S_{x}^{3}\right\rangle\right|=+\infty$ ). The dichotomy has the following geometric content. When the (even) clusters of correlated spins are tightly bound, with only a finite number of clusters having the origin in their span, then a topological argument implies that the translation symmetry has to be broken. The alternative is that the clusters of correlated spins are loosely bound, with the origin (as well as any other lattice site) belonging to the span of infinitely many correlated pairs. In that case, the above sum of the correlation function diverges. In fact, in our representation, that sum measures the number of correlated spin pairs with $x<0$ and $y>0$.

The dichotomy discussed here is reminiscent of the one found by Affleck and Lieb for the Heisenberg antiferromagnetic spin chain with half-integer spins [7]. However, unlike the dichotomy of [3], the one discussed here is not restricted to half-integer spin. The string order parameter mentioned above is also a variant of one which has been found relevant before in the context of the Heisenberg model. In fact, the method introduced here applies to quite a broad class of antiferromagnetic spin models, to be discussed in a subsequent paper [5]. 
3) Relation with the Potts models. The ground states of the $1 \mathrm{D}$ spin chains with the Hamiltonian (1.1), as well as the Gibbs states $\exp (-\beta H)$ are related to Potts model, which in case of the translation invariant interaction are always at the self-dual point. The existence of a relation was noted, at the level of a similarity of the spectra of the relevant transfer matrices, by Baxter [8] for the spin 1/2 model, and by Affleck (who introduced the general spin $S$ model with interaction $-P^{(0)}$ ), Batchelor and Barber, and Klümper $[6,11,39]$ for general spins. The representation employed here makes this relation very explicit. In particular, the dimerization corresponds to the existence of long range order in the corresponding Potts model, and the expectation values of any observable of the spin chain can be expressed in terms of quantities calculated within the Potts model. The relation presented here extends also to models with inhomogeneous couplings (for which the corresponding Potts models are no longer at their transition point), and thus extends beyond the exactly soluble cases discussed in $[8,6,11,39]$.

4) Decay rate. Using the geometric representation, and the FKG inequalities which it allows to bring to bear on the problem, we derive an effective bound on the decay of correlations of general observables in terms of the truncated two-point function $\tau(x, y)$ of an associated two-dimensional $(2 S+1)^{2}$-state Potts model:

$$
\left|\left\langle A B_{z}\right\rangle\right| \leqq C_{A} C_{B} \sum_{\substack{x \in \overline{\operatorname{supp}} A \\ y \in \operatorname{supp} B_{z}}} \tau(x, y) .
$$

For a more complete statement and the notation see Theorem 7.2. Assuming that the truncated two-point function of the two-dimensional Potts model in a magnetically ordered pure phase always has exponential decay, this result implies the existence of a spectral gap in the ground states of the spin $S$ models with interaction $-P^{(0)}$ whenever dimerization occurs. The case of staggered couplings, $\left\{J_{\text {even }}, J_{\text {odd }}\right\}$, is of interest for the discussion of the spin Peierls instability. Our method confirms the results obtained by Cross and Fisher for the exponents describing the leading behavior of the energy and the mass gap as a function of $\left|J_{\text {even }}-J_{\text {odd }}\right|[15]$.

This paper serves as an introduction to a technique of wider application, which is based on a decomposition of the Gibbs states of a large class of quantum spin Hamiltonians as superpositions of what we call quasi-states (see Appendix I). In a subsequent paper we show that such a representation exists for any isotropic nearest neighbor interaction under the condition that there are no frustration effects. No frustration essentially means that the lattice is bipartite and the Hamiltonian has ferromagnetic interactions only within each sublattice, and all interactions between spins of different sublattices are antiferromagnetic. In case there are only ferromagnetic interactions the bipartite structure is irrelevant.

For the more general case, the method used here permits to give a natural description of the occurrence of a fractional spins at the edges of finite chains. Let us just mention some results for the spin-1 antiferromagnetic chain with Hamiltonian:

$$
H=\sum_{x} a \mathbf{S}_{x} \cdot \mathbf{S}_{x+1}+b\left(\mathbf{S}_{x} \cdot \mathbf{S}_{x+1}\right)^{2}
$$

with the coupling constants $a$ and $b$ satisfying $a \geqq 0$ and $b \leqq 0$. Again there is a dichotomy [5]: under the assumption of sufficiently fast decay of correlations 
(expected to be violated only when $a=-b$ ) either

1) the ground state of the infinite system dimerizes, and thus breaks the translation invariance of the Hamiltonian,

or, 2) finite pieces of the chain behave as if near each edge there was an excess spin $S=\frac{1}{2}$.

The latter case corresponds to the Haldane phase [30] and the spin-1/2's at the edges have been observed in electron spin resonance experiments on NENP $[29,27,28]$.

\section{Quasi-State Decompositions for Equilibrium States of Quantum Spin Systems}

In this section we derive the path integral representation for the ground state, and the equilibrium states, which is employed in the derivation of the results described in the introduction. The discussion applies to a more general class of systems than those covered by Eq. (1.1). We also introduce here the notion of a "quasi-state decomposition" of a quantum state. Some of its basic properties are presented in Appendix I.

Before turning to the derivation, let us state the net result for the spin $S$ models with interaction $-P^{(0)}$.

2.1. The Functional Integral Representation for the Spin S Models with Interaction $-P^{(0)}$. Absorbing a convenient constant in its definition, the Hamiltonian is now given by:

$$
H=\sum_{x}\left\{1-(2 S+1) P_{x, x+1}^{(0)}\right\}
$$

and we are considering finite chains of spins of magnitude S. (The validity of Proposition 2.1 below is actually not restricted to the one-dimensional case, though it requires the model to be frustration free, i.e., to have a bipartite structure.)

We denote by $\sigma=\left\{\sigma_{x}\right\}$ a configuration of joint values for the commuting family of observables $\left\{S_{x}^{(3)}\right\}$. These configurations form a natural parametrization for an orthonormal basis of the Hilbert space of the system's state vectors. We obtain the following representation for the matrix elements, in this basis, of the operator $e^{-\beta H}$.

For each specified pair of configurations, $e^{-\beta H}\left(\sigma^{\prime}, \sigma\right)$ is given by an integral over various possible histories of a time dependent configuration $\sigma(t)=\left\{\sigma_{x}(t)\right\}$, with the time $t$ ranging over the interval $[0, \beta]$, and $\sigma(0)=\sigma$ and $\sigma(\beta)=\sigma^{\prime}$. What remains to be specified is the description of the configurations contributing to this integral, and the measure with which they are integrated over.

The contributing spin configurations are piecewise constant in time. When a change occurs, the spins change simultaneously at a pair of neighboring sites, but the two spins are constrained to add to zero both before and after the change. A useful description of the process is obtained by associating with each spin configuration a collection of "horizontal" bonds (in the space-time diagram in which time is in the vertical direction) linking the pairs of related sites in all the discontinuity events. For a technical reason, we find it convenient to somewhat modify this relation, and consider the bonds as enabling, rather than forcing the spin flips. (This extension yields a higher degree of independence in the measure 
seen below.) The integral over the time dependent spin configurations is based on an integral over those time indexed bonds, which we denote by the symbol $\omega$.

We shall use the following symbol for the consistency indicator function:

$\mathbf{I}[\sigma(\cdot) \mid \omega]= \begin{cases}1 & \text { if } \sigma_{x}(t) \text { satisfies the above described constraints } \\ \text { and all its discontinuities occur at bonds in } \omega \\ 0 & \text { otherwise }\end{cases}$

and $\mathbf{I}_{\text {per }}[\sigma(\cdot) \mid \omega]$ which equals one if, in addition, $\sigma(\cdot)$ is periodic in time $\left(\sigma_{x}(\beta)=\sigma_{x}(0)\right)$.

The collection of all the spin configurations which are consistent with a given bond configuration $\omega$ is conveniently described with the aid of a loop decomposition of the space-time diagram (which forms a finite-volume subset of $\mathbb{Z} \times[0, \beta]$ as illustrated in Fig. 1). The loop to which a point $(x, t)$ belongs is found by moving from it along the vertical line till a bond is reached. Upon reaching a bond, the path traverses it, and then continues in the reversed time direction along the vertical line to which it just crossed. This procedure is continued until the path either closes (by returning to its starting point), or reaches the time $t=0$ or $t=\beta$. For the timeperiodic constraint, the loops do not stop at $t=0, \beta$ but reemerge at the other end. Following these instructions, space-time is decomposed into a collection of lines which may form either closed loops or lines connecting pairs of "boundary sites" (at $t=0, \beta$ ) (in the non-periodic case). Drawing each bond in duplicate, the corresponding lines may be drawn so that they do not cross. For a specified $\omega$, the consistent spin configurations are completely characterized by the condition that the staggered spins, $(-1)^{x} \sigma_{x}(t)$, are constant along each of the loops of $\omega$. In particular, for the periodic constraint, the number of consistent spin configurations is, $(2 S+1)^{l_{\text {per }}(\omega)}$, with $l_{\text {per }}(\omega)$ being the number of loops.

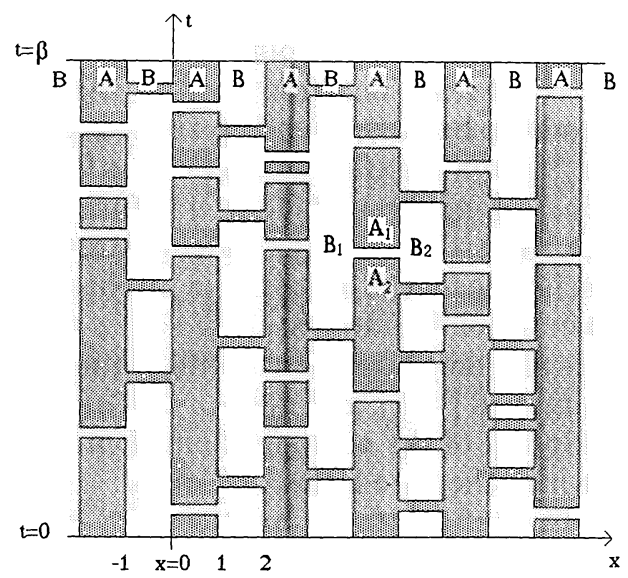

Fig. 1. A space-time configuration $\omega$ for the $-P^{(0)}$ quantum spin chain, at an inverse temperature $\beta$. As in a more general case, the spins are correlated within loops drawn by following the lines in the space-time. A special feature of this interaction is that the loops can be viewed as the boundaries of the connected clusters of two random cluster models, dual to each other. The shaded areas are the connected $A$-clusters, the connected $B$-clusters are left blank. $A_{1}, A_{2}, B_{1}, B_{2}$ are the four independent regions surrounding a bond that appear in the proof of the Euler relation. The trace of the loops on the $t=0$ line shows a decomposition of the spins into random clusters of zero spin 
The relevant measure for the time dependent spin configurations can be constructed by means of a product measure, obtained by the integration over $\omega$ with an auxiliary Poisson process distribution, $\rho_{[0, \beta]}(d \omega)$, and the discrete summation over the $(2 S+1)^{l_{\operatorname{ler}(\omega)}}$ consistent spin configurations (i.e., those with $\mathbf{I}[\sigma(\cdot) \mid \omega]=1)$. The Poisson measure is characterized by the condition that the mean bond density is 1 , and that they occur independently in disjoint regions. The contribution of a given bond configuration to the integral is enhanced by the factor $(2 S+1)^{\operatorname{lpr}(\omega)}$, and therefore its effective weight in the partition sum is given by the probability measure

$$
\mu_{\beta}(d \omega)=\left(\mathscr{Z}_{\beta}\right)^{-1} \rho_{[0, \beta]}(\mathrm{d} \omega)(2 S+1)^{l_{\operatorname{per}(\omega)}} .
$$

The situation is summarized in the following proposition.

Proposition 2.1. For a finite system with the Hamiltonian (2.1):

i)

$$
\left.\left\langle\sigma^{\prime}\left|e^{-\beta H}\right| \sigma\right\rangle=\operatorname{sign}\left(\sigma^{\prime}, \sigma\right) \int \rho_{[0, \beta]}(d \omega) \sum_{\sigma(\cdot): \mathbf{I}[\sigma(\cdot) \mid \omega]=1} \mathbf{I} \mid \sigma(\beta)=\sigma^{\prime}, \sigma(0)=\sigma\right] .
$$

ii) The partition function is given by:

$$
\mathscr{Z}_{\beta}=\operatorname{Tr} e^{\beta \sum_{x}\left((2 S+1) \boldsymbol{P}_{x, x+1}^{(0)}-1\right)}=\int \rho_{[0, \beta]}(d \omega)(2 S+1)^{l_{\operatorname{per}(\omega)}} .
$$

iii) The equilibrium expectation values of observables which are functions of the operators $S_{x}^{(3)}$ can be expressed as

$$
\frac{\operatorname{Tr} f\left(\left\{S_{x}^{3}\right\}\right) e^{-\beta H}}{\mathscr{Z}_{\beta}}=\int \mu(d \omega) E_{\omega}(f),
$$

where $\mu(d \omega)=\mathscr{Z}_{\beta}^{-1} \rho_{[0, \beta]}(d \omega)(2 S+1)^{l_{\operatorname{ler}(\omega)}}$ and the expectation functional $E_{\omega}(f)$ is obtained by averaging, with equal weights, over all the spin configurations consistent with $\omega$ :

$$
E_{\omega}(f)=\frac{1}{(2 S+1)^{l \operatorname{ler}(\omega)}} \sum_{\sigma: \mathbf{I}|\sigma(\cdot)| \omega]=1} f(\sigma(t=0)) .
$$

In the above proposition and in the rest of this paper by $\mathbf{I}[\ldots]$ we denote the indicator function of the event described between the brackets. Of course, the objects $\mathscr{Z}_{\beta}, \rho_{[0, \beta]}(d \omega), E_{\omega}(f)$ and $\mu(d \omega)$, depend on the size of the finite system and the magnitude $S$ of the spins.

The functionals $E_{\omega}$ can be extended to the full algebra of observables (see Sect. 2.5 for explicit expressions). Thus Eq. (2.6) is akin to a representation of the equilibrium state as a superposition of states. That, however, is only partially true. The linear functionals do not have the full positivity properties of quantum states. Nevertheless, this point of view is very useful, and is well justified in so far as the expectation values of the special (but important) subalgebra of observables $\left\{f\left(\left\{S_{x}^{3}\right\}\right)\right\}$ is concerned. We refer to such functionals as quasi-states. The notion is elucidated in Appendix I.

As given by Eq. (2.7), in each quasi-state $E_{\omega}$ the joint distribution of the spins takes a very simple form. In particular:

$$
E_{\omega}\left(S_{x}^{3} S_{y}^{3}\right)=(-1)^{|x-y|} C(S) \mathbf{I}[(x, 0) \text { and }(y, 0) \text { are on the same loop }]
$$

with $C(S)=\frac{1}{2 S+1} \sum_{m=-s}^{S} m^{2}=\frac{1}{3} S(S+1)$. 
Hence, the spin-spin correlation is proportional to the probability, with respect to the effective probability measure on the space of bond configurations, that two sites are on the same loop of $\omega$ :

$$
\left\langle S_{x}^{3} S_{y}^{3}\right\rangle=(-1)^{|x-y|} C(S) \operatorname{Prob}_{\mu}((x, 0) \text { and }(y, 0) \text { are on the same loop) . }
$$

The rest of this section is devoted to the derivation of Proposition 2.1, and of similar results for other systems (e.g. the Heisenberg ferromagnet). The discussion of the specific properties of the model with $H=-\sum_{x} P_{x, x+1}^{(0)}$ is resumed in Sect. 3.

2.2 Poisson Process Representation of $e^{-\beta H}$. We now turn to the derivation of the functional integral representation, which is done in the broader context of operators of the form

$$
H_{\Gamma}=-\sum_{b \in \mathscr{B}} J_{b} h_{b}
$$

where $\Gamma$ is a (finite) collection of sites, $\mathscr{B}$ is a collection of subsets of $\Gamma$, and for each $b \in \mathscr{B}, h_{b}$ is a self-adjoint operator acting in the Hilbert space $\bigotimes_{i \in b} \mathscr{H}_{i}$, with $\mathscr{H}_{i}$ the state space at the site $i$, and $J_{b}$ are non-negative coupling constants. We refer to the sets $b \in \mathscr{B}$ as bonds, although for the moment they are not required to be pairs of sites.

Thermal equilibrium states of the system, and its ground state (approached in the limit $\beta \rightarrow \infty$ ), are associated with the operator $e^{-\beta H}$. Following is a general expansion of such operators by means of integrals over a Poisson process. The symbols $\rho$ and $\omega$ appearing here are defined afresh, but their usage is consistent with the example discussed in the previous subsection.

$$
\begin{aligned}
e^{-\beta H} & =e^{\beta \sum_{b \in \mathscr{B}} J_{b}} \lim _{\Delta t \rightarrow 0}\left(\prod_{b \in \mathscr{B}} e^{\left(-J_{b}+J_{b} h_{b}\right) \Delta t}\right)^{\beta / \Delta t} \\
& =e^{\beta \sum_{b \in \mathscr{B}} J_{b}} \lim _{\Delta t \rightarrow 0}\left(\prod_{b \in \mathscr{B}}\left\{\left(1-J_{b} \Delta t\right)+J_{b} h_{b} \Delta t\right\}\right)^{\beta / \Delta t} \\
& =e^{\beta \sum_{b \in \mathscr{B}} J_{b}} \int \rho_{[0, \beta]}^{J}(\mathrm{~d} \omega) \prod_{(b, t) \in \omega}^{*} h_{b},
\end{aligned}
$$

where $\omega=\left\{\left(b_{i}, t_{i}\right)\right\} \subset \mathscr{B} \times[0, \beta]$ is a configuration of time indexed bonds, $\prod^{*}$ is the time ordered product:

$$
\prod_{b \in \omega}^{*} h_{b}=h_{b_{k}} \ldots h_{b_{2}} h_{b_{1}}, \text { such that } t_{b_{1}}<t_{b_{2}} \cdots<t_{b_{k}}
$$

and $\rho^{J}(d \omega)$ is a probability measure, under which $\omega$ forms a Poisson process over $\mathscr{B} \times[0, \beta]$, with the Poisson density $\prod_{b} J_{b} d t$. Thus, $\omega$ forms a random countable collection of time-indexed bonds which occur independently in disjoint regions of $\mathscr{B} \times[0, \beta]$.

The Poisson integral formula (2.11) offers a non-commutative version of the familiar power series expansion of the exponential function. 
2.3. The Quasi-State Decomposition. In a wide class of models there exists an orthonormal basis $\{|\alpha\rangle\}$ of the Hilbert space of the system such that for all $\omega$

$$
\left\langle\alpha\left|\prod_{(b, t) \in \omega}^{*} h_{b}\right| \alpha\right\rangle \geqq 0 .
$$

For such models the Poisson integral formula (2.11) provides a starting point for a quasi-state decomposition (Q-S decomposition) of the Gibbs state defined by

$$
\langle Q\rangle=\frac{\operatorname{Tr} e^{-\beta H} Q}{\operatorname{Tr} e^{-\beta H}} .
$$

As we shall see, the condition (2.13) can be met, in suitable bases, for both ferromagnetic and antiferromagnetic models, and it does not require the existence of a basis in which all $h_{b}$ have only non-negative matrix elements as was the case in various previous approaches $[14,24,26,33,37,44,45,51,52,53]$ (for a treatment of a much wider class of interactions see [5]).

The Q-S decomposition resulting from (2.13) takes the form:

$$
\langle\cdot\rangle=\int \mu(d \omega)\langle\cdot\rangle_{\omega}
$$

with

$$
\langle Q\rangle_{\omega}=\frac{\operatorname{Tr} K(\omega) Q}{\operatorname{Tr} K(\omega)}, \quad K(\omega)=\prod_{(b, t) \in \omega}^{*} h_{b},
$$

and

$$
\mu(d \omega)=\frac{\rho_{[0, \beta]}^{J}(d \omega) \operatorname{Tr} K(\omega)}{\int \rho_{[0, \beta]}^{J}(d \omega) \operatorname{Tr} K(\omega)} .
$$

Important features of the model are reflected in: i) the structure of the quasistates $\langle\cdot\rangle_{\omega}$, and ii) the relative weights in the decomposition (2.15) of different classes of $\omega$. To illustrate this, we now look in detail at the spin-1/2 ferromagnetic and antiferromagnetic models.

2.4. The Spin-1/2 Heisenberg Ferro- and Antiferromagnet. The Heisenberg Hamiltonian is

$$
\begin{aligned}
H^{A F} & =\stackrel{+}{(-)} \frac{1}{2} \sum_{\langle x, y\rangle} J_{x, y} \overrightarrow{\boldsymbol{\sigma}}_{x} \cdot \overrightarrow{\boldsymbol{\sigma}}_{y}+\text { Const. } \\
& =-\sum_{\langle x, y\rangle} J_{x, y} h_{\{x, y\}}^{A F}+\text { Const. }
\end{aligned}
$$

where $\overrightarrow{\boldsymbol{\sigma}}=\left(\sigma^{1}, \sigma^{2}, \sigma^{3}\right)$ are the usual Pauli matrices and the sum is over a set of pairs of sites. The signs are chosen such that we can always assume that the coupling constants $J_{x, y}$ are non-negative. The following choice of $h_{b}$ permits us to cast both the ferromagnetic and the antiferromagnetic Hamiltonians in the form of (2.10):

$$
h_{b}=\left\{\begin{array}{ll}
T_{x, y} & \text { ferromagnet } \\
2 P_{x, y}^{(0)} & \text { antiferromagnet }
\end{array},\right.
$$

where $T_{x, y}$ and $P_{x, y}^{(0)}$ are the transposition and singlet-projection operators acting in the Hilbert space of the sites $x$ and $y$ (so, $T_{x, y}$ interchanges the states at the sites 
$x$ and $y$ ). Use is made here of the relations

$$
\overrightarrow{\boldsymbol{\sigma}}_{x} \cdot \overrightarrow{\boldsymbol{\sigma}}_{y}=2 T_{x, y}-1=1-4 P_{x, y}^{(0)} .
$$

In discussing the matrix elements

$$
\left\langle\sigma\left|\prod_{b \in \omega}^{*} h_{b}\right| \sigma^{\prime}\right\rangle=\sum_{\sigma_{t_{1}}, \ldots, \sigma_{t_{k-1}}}\left\langle\sigma\left|h_{b_{1}}\right| \sigma_{t_{1}}\right\rangle\left\langle\sigma_{t_{1}}\left|h_{b_{2}}\right| \sigma_{t_{2}}\right\rangle \ldots\left\langle\sigma_{t_{k-1}}\left|h_{b_{k}}\right| \sigma^{\prime}\right\rangle,
$$

it is convenient to consider a space time picture in which the RHS is viewed as a sum over paths in the spin configuration space, with $\left\{\sigma_{x}^{3}\right\}$ defined at all times. That configuration is piecewise constant, and the amplitude for the process is determined by the matrix elements of the operators $\left\{h_{b}\right\}$. Beyond this point the two cases need be discussed separately.

2.4.a: The ferromagnet $\left(H^{F}\right)$. In the ferromagnetic case, the $h_{b}$ are transpositions, which occur with amplitude 1 . Thus, the time-ordered product (2.12) consists of a sequence of transpositions which result in a permutation which we denote $\pi(\omega)$. In particular, it is easily seen that

$$
\operatorname{Tr} K(\omega)=2^{l^{F}(\omega)},
$$

where $l^{F}(\omega)$ is the number of cycles in the corresponding permutation. (The factor 2 reflects the dimension of the single-site Hilbert space.) More generally, quantities of the form $\operatorname{Tr} f\left(\left\{\sigma_{x}^{3}\right\}\right) e^{-\beta H^{F}}$ are described by the following construction - which is similar to but not quite the same as the one presented in Sect. 2.1 for the Hamiltonian considered there.

The paths $\sigma(t)$ which contribute are constrained, by the nature of the trace, to have $\sigma(t=0)=\sigma(t=\beta)$, and thus are periodic in time. The configuration $\omega$ is visualised, in a space-time graph, by a collection of "horizontal" bonds connecting "vertical" lines indexed by the lattice sites. For each $\omega$, the contributing spin configurations are obtained by decomposing the graph into a collection of loops. The ferromagnetic loops are obtained by replacing the antiferromagnetic bonds in Fig. 1 by ferromagnetic ones (see Fig. 2). The loop to which a point $(x, t)$ belongs is found by moving "upward" along the vertical line at that point till a bond is reached. At a ferromagnetic bond $b=\{x, y\}$, the path crosses from $x$ to $y$ and continues in the positive time direction. This procedure is continued until the time $t=\beta$ is reached at which point the path jumps to $t=0$ at the same site, i.e. $t=0$ and $t=\beta$ are identified. The process is repeated until one comes back at the point $(x, t)$.

The permutation $K(\omega)$ and its cycle decomposition is easily read from the above picture: $K(\omega)$ takes $x$ into the site where the loop drawn starting from $(x, 0)$ returns, for the first time, to $t=0$.

The discussion in Sect. 2.3 yields now the following proposition.
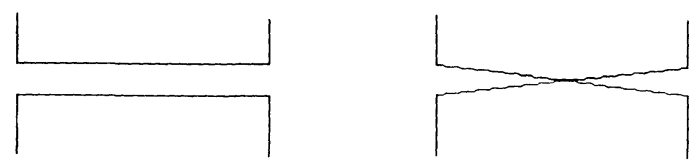

Fig. 2. The graphical representation of the ferro- and antiferromagnetic bonds used in the drawing of the loop configuration $\omega$ 
Proposition 2.2 For the spin- $\frac{1}{2}$ Heisenberg ferromagnet, the partition function and the expectation values of observables generated by $\left\{\sigma_{x}^{3}\right\}_{x \in \Gamma}$ are given by:

$$
\mathscr{Z}_{\beta}^{F}=\operatorname{Tr} e^{\beta} \sum_{\langle x, y\rangle} J_{x, y}\left(T_{x, y}-1\right)=\int \rho_{[0, \beta]}^{J}(d \omega) 2^{l^{F}(\omega)},
$$

and

$$
\frac{\operatorname{Tr} f\left(\sigma^{3}\right) e^{-\beta H^{F}}}{\mathscr{Z}_{\beta}^{F}}=\int \mu_{F}(d \omega) E_{\omega}^{F}(f),
$$

where $\mu^{F}(d \omega)$ is the probability measure

$$
\mu^{F}(d \omega)=\left(\mathscr{Z}_{\beta}^{F}\right)^{-1} \rho_{[0, \beta]}^{J}(d \omega) 2^{l^{F}(\omega)}
$$

and the expectation functional $E_{\omega}^{F}(f)$ is obtained by averaging, with equal weights, over all the spins configurations which take common values $\left(\eta_{\nu}= \pm 1\right)$ on the cycles $\{\gamma\} \in \omega$, corresponding to $K(\omega)$ :

$$
E_{\omega}^{F}(f)=2^{-l^{F}(\omega)} \sum_{\eta_{\gamma}= \pm 1} f(\sigma(\eta))
$$

In fact, $E_{\omega}^{F}(f)$ are quasi-states (adapted to the algebra generated by $\left\{\sigma_{x}^{3}\right\}_{x \in \Gamma}$ according to the definition in Appendix I). In Sect. 2.5, below, we shall discuss the expectation values of other observables. However, let us note here that two interesting choices for $f$ are: $f_{1}\left(\sigma^{3}\right)=\sigma_{x}^{3} \sigma_{y}^{3}$ and $f_{2}\left(\sigma^{3}\right)=\exp \left(\beta h \sum_{x \in \Gamma} \sigma_{x}^{3}\right)$. Here, in contrast to our overall convention, $\sigma_{x}^{3}$ denotes a Pauli matrix with eigenvalues \pm 1 . For these functions $E_{\omega}^{F}(f)$ is given by

$$
\begin{aligned}
& E_{\omega}^{F}\left(f_{1}\right)=\mathbf{I}[(x, 0) \text { and }(y, 0) \text { are on the same loop }], \\
& E_{\omega}^{F}\left(f_{2}\right)=\prod_{\gamma \in \omega} \cosh (|\gamma| \beta h),
\end{aligned}
$$

where $|\gamma|$ denotes the number of times $\gamma$ intersects the $t=0$ axis (so, $|\gamma|=$ the length of the cycle $\pi_{\gamma}$ ). In fact very similar expressions to the ones above can be derived also for itinerant electron models. The analogue of formula (2.29) for the Hubbard model was used by Aizenman and Lieb in [3] to derive a generalization of Nagaoka's Theorem to finite temperatures.

2.4.b: The antiferromagnet $\left(H^{A F}\right)$. A significant difference between the ferromagnetic interaction and the antiferromagnetic one, which is seen already at the classical level, is the possibility of "frustration." Our analysis is restricted to the frustration-free case, which is characterized by the existence of a bipartite structure: the lattice $\Gamma$ decomposes into two sublattices, $\Gamma_{A}$ and $\Gamma_{B}$, with the couplings between two sites restricted to be antiferromagnetic if the sites belong to distinct sublattices, and, in more elaborate models in which both kinds of interactions are present (see [5]), ferromagnetic within each sublattice. For convenience we also define

$$
(-1)^{|x-y|}=\left\{\begin{array}{ll}
+1 & \text { if } x \text { and } y \text { belong to the same sublattice } \\
-1 & \text { if } x \text { and } y \text { belong to distinct sublattices }
\end{array} .\right.
$$

As we shall see now, under the assumption of a bipartite structure, the positivity condition (2.13) is satisfied even though not all the matrix elements of the operators $K(\omega)$ are positive. 
In the computation of quantities of the form $\operatorname{Tr} f\left(\left\{\sigma_{x}^{3}\right\}\right) e^{-\beta H^{A F}}$ we need the matrix elements of $h_{b}$ for the antiferromagnet:

$$
h_{b}=2 P_{b}^{(0)}=\sum_{\alpha, \beta \in\left\{\frac{1}{2},-\frac{1}{2}\right\}}(-1)^{\alpha-\beta}|\beta,-\beta\rangle\langle\alpha,-\alpha| .
$$

Note the similarity between (2.31) and (1.2).

At this point we have a choice: the spin $1 / 2 \mathrm{AF}$ system can be discussed in a form close to that of the spin $1 / 2$ ferromagnet or in a form which is suitable for the more general spin-S models (with the Hamiltonian (2.1)). In order to lay the grounds for a sequel to this paper, where we encounter spin $1 / 2$ systems with mixed $\mathrm{F}$ and AF interactions, we shall present the first option before treating the general spin case.

As in the ferromagnetic case, for a given $\omega$ the allowed time dependent spin configurations $\left\{\sigma_{x}^{3}\right\}$ are piecewise constant (in time) and can change only at pairs of sites where a bond occurs. When a change occurs it is again a transposition. However, there are the following differences from the ferromagnetic case:

i) there is a restriction that where a bond occurs the two spins add up to zero (both before and after the event),

ii) at a given bond a transposition may or may not occur,

iii) the amplitude for a given "path" is $(-1)$ raised to the number of transpositions, i.e. it equals the parity of the resulting permutation.

Lemma 2.3. In a bipartite system, for each pair of configurations $\left\{\sigma, \sigma^{\prime}\right\}$, all the permutations which take $\sigma$ into $\sigma^{\prime}$ and which can be written as products of transpositions exchanging sites on different sublattices have a common parity, denoted here $\operatorname{sign}\left(\sigma, \sigma^{\prime}\right)$, with

$$
\operatorname{sign}(\sigma, \sigma)=+1
$$

Proof. Each transposition changes the number of positive spins on the A-sublattice by \pm 1 . Therefore, noting that the spin configurations take values $\pm \frac{1}{2}$, the parity of any admissible permutation is

$$
(-1)^{\sum_{x \in r_{1}}\left(\sigma_{x}-\sigma_{x}^{\prime}\right)}
$$

We now derive the following formula for the matrix elements of the operators $K(\omega)$ :

$$
\begin{aligned}
\left\langle\sigma\left|\prod_{b \in \omega}^{*} h_{b}\right| \sigma^{\prime}\right\rangle & =\sum_{\sigma_{t_{1}}, \ldots, \sigma_{t_{k-1}}}\left\langle\sigma\left|h_{b_{1}}\right| \sigma_{t_{1}}\right\rangle\left\langle\sigma_{t_{1}}\left|h_{b_{2}}\right| \sigma_{t_{2}}\right\rangle \cdots\left\langle\sigma_{t_{k-1}}\left|h_{b_{k}}\right| \sigma^{\prime}\right\rangle \\
& =\operatorname{sign}\left(\sigma, \sigma^{\prime}\right) 2^{l_{0}^{A F}(\omega)} \mathbf{I}_{A F}\left[\sigma, \sigma^{\prime} \mid \omega\right],
\end{aligned}
$$

where $l_{0}^{A F}(\omega)$ and $\mathrm{I}_{A F}\left[\sigma, \sigma^{\prime} ; \omega\right]$ are defined graphically, in a decomposition of the spacetime graph into paths, which in many ways is similar to the one seen in the ferromagnetic case. The paths now come in two kinds: open paths with end points of the form $(x, 0)$ and $(x, \beta)$, and closed paths which we call internal loops (see Fig. 1). The open paths are constructed by starting from any point $(x, t)$ with $x \in \Gamma$ and $t=0$ or $\beta$, by moving in the vertical direction until a bond is met. Upon the traversal of a bond, the orientation of the motion in time is reversed. The path stops upon reaching $t=0$ or $t=\beta$. The internal loops are obtained in the same way, starting 
from any point $(x, t) \in \Gamma \times[0, \beta]$ that does not already belong to an open path and a loop is completed when the path comes back to its starting point. $l_{0}^{A F}(\omega)$ denotes the number of internal loops. $\mathrm{I}_{A F}\left[\sigma, \sigma^{\prime} ; \omega\right]$ is defined in terms of the open paths which we interpret as imposing a pairing condition on the spin configurations $\sigma=\sigma(0)$ and $\sigma^{\prime}=\sigma(\beta)$. The condition is that for any pair of points $\mathbf{x}=(x, t)$ and $\mathbf{y}=\left(y, t^{\prime}\right)$ with $t$ and $t^{\prime}$ either 0 or $\beta$, that are the end points of a path in $\omega$, one has

$$
\sigma(t)_{x} \sigma\left(t^{\prime}\right)_{y}=(-1)^{|x-y|}=1-2 \delta_{t, t^{\prime}}
$$

$\mathrm{I}_{A F}\left[\sigma, \sigma^{\prime} \mid \omega\right]=1$ if (2.35) is satisfied for all open paths in $\omega$ and 0 otherwise.

Formula (2.34) is now a direct consequence of the graphical representation of $\omega$ and the definitions given above.

When computing $\operatorname{Tr} K(\omega)$ one identifies $t=0$ and $t=\beta$. Then, all paths are closed, i.e. they are loops, and we write $l^{A F}(\omega)$ or $l_{\text {per }}(\omega)$ to denote the total number of loops in $\omega$.

The above discussion leads now directly to the representation given by Proposition 2.1 for $S=1 / 2$.

2.5. Structure of the Quasi-States. From the expressions in Proposition 2.1 and 2.2 it is obvious that the quasi-states $\langle\cdot\rangle_{\omega}$ depend only on the structural properties of $\omega$ revealed in the random loop picture of the configuration. In fact, the only relevant property of $\omega$ is how its random loops link together sets of sites at $t=0$. In both the ferro- and the antiferromagnet a quasi-state $E_{\omega}$ is uniquely determined by the permutation $\pi_{\omega}$ of the sites in $\Gamma$, which takes the site $x \in \Gamma$ to the site $\pi(x)$ which is where the loop at $x$, starting off in the positive time direction, intersects $t=0$ for the next time. There is a one-to-one correspondence between the cycles in $\pi_{\omega}$ and the loops in $\omega$ that intersect the $t=0$ hyperplane. Let $\gamma_{0}$ denote such a generic cycle:

$$
\gamma_{0}=\left(\begin{array}{ccccc}
x_{1} & x_{2} & \ldots & x_{r-1} & x_{r} \\
x_{2} & x_{3} & \ldots & x_{r} & x_{1}
\end{array}\right) .
$$

A first observation to make is then that in the functional $E_{\omega}^{\#}$, with $\#=F$ or $A F$, there are no correlations between the spins on two sets of sites which support distinct cycles in the permutation:

$$
E_{\omega}^{\#}\left(\prod_{\gamma_{0}} A_{\gamma_{0}}\right)=\prod_{\gamma_{0}} E_{\omega}^{\#}\left(A_{\gamma_{0}}\right),
$$

where $A_{\gamma_{0}}$ is an arbitrary operator acting on the sites $\left\{x_{1}, \ldots, x_{r}\right\}$. Moreover, $E_{\omega}^{\#}\left(A_{\gamma_{0}}\right)$ depends on $\omega$ only through $\gamma_{0}$ and we therefore might as well denote it by $E_{\gamma_{0}}^{\#}\left(A_{\gamma_{0}}\right)$.

As the $E_{\omega}^{\#}$ are linear, they are completely determined by their values on operators of the form $A_{\gamma_{0}}=\sigma_{x_{1}}^{i_{1}} \cdots \sigma_{x_{r}}^{i_{r}}$, where $i_{j} \in\{0,1,2,3\}$, and by $\sigma^{0}$ we denote the identity operator. It is a straightforward exercise to compute $E_{\gamma_{0}}^{\#}\left(\sigma_{x_{1}}^{i_{1}} \ldots \sigma_{x_{r}}^{i_{r}}\right)$ starting from the space-time picture: we consider the spin to be a piecewise constant function along $\gamma_{0}$ taking values $\sigma^{3}= \pm 1$. Where there is a factor $\sigma^{3}$ the expectation value picks up a factor \pm 1 according to what the spin is at that point. $\sigma^{1}$ reverses the spin and $\sigma^{2}=i \sigma^{1} \sigma^{3}$. For the ferromagnet the result is:

$$
E_{\gamma_{0}}^{F}\left(\sigma_{x_{1}}^{i_{1}} \ldots \sigma_{x_{r}}^{i_{r}}\right)=\frac{1}{2}\left\{\left\langle\frac{1}{2}\left|\sigma^{i_{1}} \ldots \sigma^{i_{r}}\right| \frac{1}{2}\right\rangle+\left\langle-\frac{1}{2}\left|\sigma^{i_{1}} \ldots \sigma^{i_{r}}\right|-\frac{1}{2}\right\rangle\right\} .
$$


Classically, the antiferromagnetic state is equivalent to a ferromagnetic one of the staggered spins

$$
\hat{\sigma}_{x}=(-1)^{x} \sigma_{x} .
$$

We find that such a relation holds between the two quasi-states corresponding to the same permutation $\gamma_{0}$. The relation (2.39) does of course not correspond to a unitary transformation of the full algebra of observables of the system and is possible only because we are not dealing with true quantum states but only with quasi-states. A compact expression for the antiferromagnetic functionals is:

$$
E_{\gamma_{0}}^{A F}\left(\sigma_{x_{1}}^{i_{1}} \cdots \sigma_{x_{r}}^{i_{r}}\right)=E_{\gamma_{0}}^{F}\left(\hat{\sigma}_{x_{1}}^{i_{1}} \cdots \hat{\sigma}_{x_{r}}^{i_{r}}\right)=(-1)^{\#\{x,\} \cap \Gamma_{1}} E_{\gamma_{0}}^{F}\left(\sigma_{x_{1}}^{i_{1}} \cdots \sigma_{x_{r}}^{i_{r}}\right) .
$$

We conclude this section by summarizing the results of Propositions 2.1 and 2.2 in the following way: we found a representation of the ferro- and antiferromagnetic ground states as a convex combination of quasi-states which for the ferromagnet are a partition of the lattice into subsets on which the spins are locked together in a parallel state. For the antiferromagnet the spins are rigidly correlated in a staggered manner. This picture can be considered as a generalization of the VBS-states where neigboring spins are paired into the singlet state (for a different generalization see [17]). In particular, depending on the properties of $\mu(d \omega)$, the states can also have long range order, characterized by the fact that the clusters percolate throughout the system. Finally we want to remark that the quasi-states each have a much larger symmetry group (which depends on $\omega$ ) than the ground state itself. Because there are no correlations between the sets of sites belonging to different loops, the spins on the distinct cycles in $\pi_{\omega}$ can be rotated independently.

2.6. The $\mathrm{SU}(2 S+1)$-Invariant Spin-S Models with Interaction $-P^{(0)}$. We now turn to the Q-S decomposition of a generalization of the spin- $-\frac{1}{2}$ Heisenberg ferro- and antiferromagnet to arbitrary values of the spin. In particular this generalization includes the one-dimensional antiferromagnets that are the main subject of this paper. Starting from the Heisenberg Hamiltonians as they were written in Sect. 2.4, we just replace $T_{x, y}$ and $2 P_{x, y}^{(0)}$ by the corresponding operators for a spin $S$ system: in the "ferromagnetic" case the interaction $h_{b}^{F}=T_{x, y}$ interchanges the states at the sites $x$ and $y$ and for the antiferromagnet $h_{b}^{A F}=(2 S+1) P_{x, y}^{(0)}$, where $P^{(0)}$ is given in (1.2). It is obvious that both $h_{b}^{F}$ and $h_{b}^{A F}$ are $\mathrm{SU}(2)$-invariant. Due to the invariance of this interaction under parity preserving relabelings of the $2 S+1$ states, this $S U(2)$ symmetry is actually embedded in a larger (for $S \geqq 1) \mathrm{SU}(2 S+1)$-symmetry. The model with interaction $h_{b}^{F}$ is a degenerate ferromagnet and its ground states are given by all permutation symmetric states of the system. (The integrability of the one-dimensional models with interactions $h_{b}^{F}$ and $-h_{b}^{F}$ was demonstrated in the well-known work of Sutherland [50]). The interaction $h_{b}^{A F}$ is much more interesting and is the actual subject of the rest of the paper. In the case of the one-dimensional lattice, we recover the $\mathrm{SU}(2 S+1)$-invariant model that was first studied by Affleck [6] and also by Klümper [39] and Batchelor and Barber [11].

As before, we can only deal with the non-frustrated case, and let $\Gamma_{A}$ and $\Gamma_{B}$ denote the two sublattices defining the bipartite structure of the system. The $\mathrm{SU}(2 S+1)$-symmetry of the Hamiltonian with interaction (1.2) is then represented by the fundamental representation on one sublattice and the antifundamental representation on the other sublattice. 
The matrix elements of $e^{-\beta H}$ and the partition function for this model can be expressed using the same correspondence between configurations $\omega$ and sets of loops as for the spin $-1 / 2$ antiferromagnet. But now each loop has to be decorated with a label $\alpha$ taking the $2 S+1$ values $-S,-S+1, \ldots S-1, S$. The spin configuration as a function of $x \in \Gamma$ and $t \in[0, \beta]$, is now the following:

$$
S^{3}(x, t)=\left\{\begin{array}{rl}
\alpha & \text { if the label of the loop is } \alpha \text { and } x \in \Gamma_{A} \\
-\alpha & \text { if the label of the loop is } \alpha \text { and } x \in \Gamma_{B}
\end{array} .\right.
$$

The proof of Proposition 2.1 now follows by the same arguments of Sect. 2.4, taking into account the correspondence between loop labelings and space-time spin configurations given in (2.41).

In the one-dimensional case the loops can be interpreted as the boundaries of the elements in a partition of the two-dimensional space-time. As we will see in the next section, the weights with which these partitions occur are given by the Gibbs weight for the associated configurations of a Potts model at the selfdual point. This will enable us to analyse the possible long-range order in the ground state of these models.

\section{Equivalence with the Two-Dimensional $(2 S+1)^{2}$-State Potts Models}

It will be natural to consider the spin chain with a priori different coupling strengths for the even and odd bonds. More generally, we are concerned with the Hamiltonian for a spin- $S$ chain given by:

$$
H_{\left[L_{-}, L_{+}\right]}^{A F}=-\sum_{x=L_{-}}^{L_{+}-1} J_{x} h_{\{x, x+1\}}^{A F}
$$

where $h^{A F}=(2 S+1) P^{(0)}$ is defined in (1.2), $J_{x}>0$ and $L_{-}, L_{+} \in \mathbb{Z}, L_{-} \leqq 0<L_{+}$.

We shall now show that associated to the geometric structure of Sect. 2 is a Potts model, or rather a pair of dual Potts models (the $A$ - and the $B$-model). In the translation invariant case $\left(J_{x}=J\right.$ for all $\left.x\right)$ one arrives at the Potts model at its self-dual point, where it is exactly solvable [8]. In this situation the equivalence was conjectured by Affleck and established on the level of the spectrum of the transfer matrices by Batchelor and Barber [11] and Klümper [38, 39].

The Potts models are defined over a $1+1$ dimensional lattice, $\mathbb{Z} \times \mathbb{R}$, in which one of the directions (corresponding to the "time" of Sect. 2) is continuous.

To introduce the lattices on which the $A$ - and $B$-Potts model variables reside, we start from the space-time of the quantum spin chain embedded in $\mathbb{R}^{2}$ and partition $\mathbb{R}^{2}$ into vertical strips of width 1 which we label alternatingly $A$ and $B$, with the strip $0<x<1$ getting the label $A$, as in Fig. 1 . The Potts variables of the $A$-model reside on the vertical lines bisecting the $A$-strips and the variables of the $B$-model are situated on the lines bisecting the $B$-strips.

For a volume $[0, T] \times\left[L_{-}, L_{+}\right]$, the Potts configurations of the $A$-model are functions $\xi(x, t), x=$ even $+\frac{1}{2} \in\left[L_{-}, L_{+}\right]$, which are piecewise constant in time and take values in $\{1, \ldots, q\}$. In the time direction we always take the periodic boundary conditions. Other than that, there are two natural boundary conditions for a Potts model, which are exchanged under the standard duality map: the free and the wired, the latter corresponding to adding an extra strip to the left and to the right of the volume where the spins are required to assume a common value 
(we assume the convention that the partition function includes also the sum over this common value). As will emerge from our discussion, the relevant boundary conditions here depend on the label of the strip along the boundary. If it is $A$ then the $A$-model gets the free b.c. and the $B$-model the wired one, and otherwise it is the other way around.

The partition function of the $A$-model (with the relevant boundary terms) is given by

$$
\mathscr{Z}_{A}^{\text {Potts }}=\int \rho_{A}^{J^{V}}(d \omega) \sum_{\xi}^{\omega} \exp \left\{\sum_{\substack{x=\text { even }+\frac{1}{2} \\ L_{-}-1 \leqq x \leqq L_{+}}} \int_{0}^{T} d t J_{x}^{H}\left(\delta_{\xi(x, t), \xi(x+2, t)}-1\right)\right\},
$$

where $J^{V}=\left\{J_{x}^{V}\right\}$ and $\left\{J_{x}^{H}\right\}$ are sets of positive constants (these are the ferromagnetic coupling constants in the vertical and horizontal direction respectively and in which we have absorbed the inverse temperature of the Potts model), $\rho_{A}^{J^{V}}$ is a product of independent Poisson point processes on the lines $\left\{x=2 n+\frac{1}{2}\right\} \times[0, T]$ with intensity $J_{x}^{V}$, and $\sum^{\omega}$ denotes the sum over all configurations $\xi$ for which the discontinuities happen only at points $(x, t)$ in the configuration $\omega$ of the Poisson process. The sum in (3.2) should be interpreted as incorporating the boundary condition convention explained in the previous paragraph.

The Poisson measure incorporates the interaction in the vertical direction, and it can be arrived at by way of a continuum limit of ordinary discrete Potts models with vertical couplings analogous to those seen in (3.2) for the horizontal interaction.

For the $B$-model we have an analogous expression with the condition $\left\{x=\right.$ even $\left.+\frac{1}{2}\right\}$ replaced by $\left\{x=\right.$ odd $\left.+\frac{1}{2}\right\}$.

Potts models are conveniently studied via an embedding in the random cluster model formulated by Fortuin and Kasteleyn [19]. As we shall see, it is at that level that the correspondence with the quantum spin chain is most explicit. The theorem below and Theorem 7.2 present some key results which are derived by that route.

Theorem 3.1. Let the parameters of the finite quantum spin chain with interaction $-P^{(0)}$ on the interval $\left[L_{-}, L_{+}\right]$, and the ones of the $A$ - and $B$-Potts models be related as follows:

Then

$$
\begin{aligned}
J_{x}^{H} & =(2 S+1)_{x+\frac{1}{2}}^{J}, \quad J_{x}^{V}=(2 S+1)^{-1} J_{x-\frac{1}{2}}, \\
q & =(2 S+1)^{2}, \quad \beta=T .
\end{aligned}
$$

i)

$$
\begin{aligned}
-\frac{1}{\beta} \ln \mathscr{Z}^{(S)=} & -\frac{1}{T} \ln \mathscr{Z}_{A}^{\text {Potts }}-\frac{2 S}{2 S+1} \sum_{\substack{x=\text { even } \\
L_{-} \leqq x \leqq L_{+}-1}} J_{x}+2 S \sum_{\substack{x=\text { odd } \\
L_{-} \leqq x \leqq L_{+}-1}} J_{x} \\
& -\frac{1}{T}\left(\frac{1}{2}\left\{(-1)^{L_{-}}-(-1)^{L_{+}}\right\}-1\right) \ln (2 S+1) .
\end{aligned}
$$

In particular the ground state energy per site of the quantum chain equals the free energy per unit volume of the associated Potts model up to a trivial constant.

ii) The Potts model has periodic boundary conditions in the vertical direction and free boundary conditions in the horizontal direction.

iii) The distribution $\mu(d \omega)$ of the random loop representation for the quantum chain as described in Sect. 2, is identical to the distribution of the boundaries of the connected clusters of the Potts model in the FK-representation (see below). 
It should be noted that the coupling constants of both the $A$ and the $B$ Potts model depend on all the coupling constants $\left\{J_{x}\right\}$ of the quantum spin chain. The relevant coupling constants as defined in Theorem 3.1 are $J_{x}^{V}$ and $J_{x}^{H}$ with $x=$ even $+\frac{1}{2}$ for $A$-model, and $x=$ odd $+\frac{1}{2}$ for the $B$-model.

In Sect. 7 we present some explicit relations between the correlation functions of the quantum chain and those of the Potts models, which, in particular, imply a non-perturbative result on the spectral gap in the quantum spin chains with staggered couplings (see Theorem 7.1).

We now turn to the proof of Theorem 3.1.

First we derive the Fortuin-Kasteleyn representation $[18,19]$ of the twodimensional Potts models described above, by showing that the partition function and the probability measure of the Potts model are equal to the ones of a random cluster model. The random cluster model is obtained by considering a configuration $\omega=\omega_{A} \times \omega_{B}$ generated by the Poisson process $\rho_{A}^{J^{V}}\left(d \omega_{A}\right) \rho_{B}^{J^{H}}\left(d \omega_{B}\right)$, as a partition of the union of the vertical strips of type $A$ in the following way: a point $(x, t) \in \omega_{A}$ is considered as cutting the vertical strip with coordinate $x$ at the height $t$ and a point $(x, t) \in \omega_{B}$ connects the two $A$-strips adjacent to the $B$-strip at coordinate $x$ and height $t$ (see Fig. 1). The partition function of the random cluster (RC) model is then given by:

$$
\mathscr{Z}^{\mathrm{RC}}=\int \rho_{A}^{J^{V}}\left(d \omega_{A}\right) \rho_{B}^{J^{H}}\left(d \omega_{B}\right) q^{C_{A}\left(\omega_{A} \times \omega_{B}\right)},
$$

where $C_{A}\left(\omega_{A} \times \omega_{B}\right)$ denotes the number of connected clusters (consisting of pieces of strips of type $A$ ) in the configuration $\omega_{A} \times \omega_{B}$. The equality of $\mathscr{Z}^{\text {Potts }}$ and $\mathscr{Z}^{\mathrm{RC}}$ can be derived straightforwardly by expanding the exponential in (3.2):

$$
\begin{aligned}
\mathscr{Z}_{A}^{\text {Potts }}= & \int \rho_{A}^{J^{V}}(d \omega) \sum_{\xi}^{\omega_{A}} \exp \left\{\sum_{x=2 n+\frac{1}{2}} \int d t J_{x}^{H}\left(\delta_{\xi(x, t), \xi(x+2, t)-1)}\right\}\right. \\
= & \int \rho_{A}^{J^{V}}(d \omega) \sum_{\xi}^{\omega_{A}} \prod_{x} \sum_{n_{x}=0}^{\infty} \frac{1}{n_{x} !}\left(J_{x}^{H}\right)^{n_{x}} e^{-\beta J_{\lambda}^{H} n_{x}} \\
& \times \int d t_{1}^{(x)} \cdots d t_{n_{x}}^{(x)} \delta_{\xi\left(x, t_{1}^{(x)}\right), \xi\left(x+2, t_{1}^{(x)}\right)} \cdots \delta_{\xi\left(x, t_{n_{x}}^{(x)}\right), \xi\left(x+2, t_{n_{x}}^{(x)}\right)} \\
= & \int \rho_{A}^{J^{V}}\left(d \omega_{A}\right) \rho_{B}^{J^{H}}\left(d \omega_{B}\right) \sum_{\xi}^{\omega_{A}} \prod_{(y, t) \in \omega_{B}} \delta_{\xi(y, t), \xi(y+2, t)} \\
= & \int \rho_{A}^{J^{V}}\left(d \omega_{A}\right) \rho_{B}^{J^{H}}\left(d \omega_{B}\right) q^{C_{A}\left(\omega_{A} \times \omega_{B}\right)}=\mathscr{Z}^{\mathrm{RC}} .
\end{aligned}
$$

In the sequel we shall write $\omega$ for $\omega_{A} \times \omega_{B}$ and $\rho^{J}(d \omega)$ or $\rho^{\left(J^{V}, J^{H}\right)}(d \omega)$ instead of $\rho_{A}^{J^{V}}\left(d \omega_{A}\right) \rho_{B}^{J^{H}}\left(d \omega_{B}\right)$.

A by now standard and very convenient tool for the study of the random cluster model is provided by the FKG inequalities [20]. Following is the FKG-structure which will be used to derive some of the main results of this paper in Sects. 4-7.

We define a partial order on the configurations $\omega$ as follows: $\omega^{\prime} \leq \omega$ if the set of $A$-bonds in $\omega$ is contained in the set of $A$-bonds in $\omega^{\prime}$ and the set of $B$-bonds in $\omega$ contains the set of $B$-bonds in $\omega^{\prime}$. (By $A$-bonds we mean bonds occurring in an $A$-strip and analogously for $B$-bonds.) As before, let $l_{\text {per }}(\omega)$ denote the number of loops in $\omega$, considered with periodic boundary conditions in time. We consider the loops as the boundaries of a collection of connected subsets (connected clusters) of the plane. Each such connected set consists of the vertical strips $n<x<n+1$ 
connected by horizontal bridges. All strips in a given cluster are either of $A$ or of $B$ type. Let $C_{A}(\omega)$ denote the number of connected clusters of $A$ type and $C_{B}(\omega)$ the number of clusters of $B$ type. With these definitions one then has the following obvious but crucial properties: $N_{A}(\omega)$ and $C_{A}(\omega)$ are decreasing functions of $\omega$ and $N_{B}(\omega)$ and $C_{B}(\omega)$ are increasing, where $N_{C}(\omega)$ denotes the number of bonds in $\omega$ which occur in the strips of type $C, C=A, B$.

In the following proposition and throughout the rest of the paper, $\rho^{J}(d \omega)$ denotes the Poisson measure on the configurations $\omega$ for the quantum spin chain on a finite interval, $\left[L_{-}, L_{+}\right]$, containing $L_{+}-L_{-}+1$ sites, and at inverse temperature $\beta$ (see Sect. 2.1). $J$ stands for the collection of coupling constants $\left\{J_{x}\right\}_{L_{-} \leqq x \leqq L}$. which determine the intensities of the independent Poisson measures for each bond. We will sometimes need to distinguish between the coupling constants for even and odd $x$; we then use the notation: $J=\left(J_{\text {even }}, J_{\text {odd }}\right)$.

One then has the following relations:

Proposition 3.2. With boundary conditions described above, the following relations hold: for any $u>0$,

where

$$
\begin{aligned}
\rho^{\left(J_{\text {even }}, J_{\text {odd }}\right)}(d \omega) u^{l_{\text {per }}(\omega)} & =c_{1} \rho^{\left(J_{\text {even }}, J_{\text {odd }}\right)}(d \omega) u^{C_{A}(\omega)+C_{B}(\omega)} \\
& =c_{2} \rho^{\left(u^{-1} J_{\text {even }}, u J_{\text {odd }}\right)}(d \omega) u^{2 C_{A}(\omega)} \\
& =c_{3} \rho^{\left(u J_{\text {even }}, u^{-1} J_{\text {odd }}\right)}(d \omega) u^{2 C_{B}(\omega)},
\end{aligned}
$$

$$
\begin{aligned}
& c_{1}=u^{-1}, \\
& c_{2}=u^{\frac{1}{1}\left\{(-1)^{L-}-(-1)^{L+}\right\}-1} \exp \left\{\beta\left(\left(1-u^{-1}\right) \sum_{x \text { even }} J_{x}+(1-u) \sum_{x \text { odd }} J_{x}\right)\right\}, \\
& c_{3}=u^{-\frac{1}{2}\left\{(-1)^{L-}-(-1)^{L+}-1\right.} \exp \left\{\beta\left((1-u) \sum_{x \text { even }} J_{x}+\left(1-u^{-1}\right) \sum_{x \text { odd }} J_{x}\right)\right\} .
\end{aligned}
$$

Proof. We start by reformulating the random loop picture as follows. As before we consider the loops as being embedded in the plane which we have divided into vertical strips labeled alternatingly $A$ and $B$ as in Fig. 1. We can then associate with each configuration $\omega$, a set of $A$ - and $B$-clusters as follows. When $\omega$ contains an activated bond in an $A$-strip this bond is considered as forming a connection between the two $B$-strips to the immediate left and right of it and as cutting through the $A$-strip to which it belongs, and analogously for the bonds in a $B$-strip. Thus for each configuration $\omega$ we have obtained a collection of clusters of $A$ and $B$ type and such that two different clusters with a piece of common border are of different type. $C_{A}(\omega)$ and $C_{B}(\omega)$ are the number of $A$-clusters and $B$-clusters in $\omega$, respectively.

We consider a finite interval of the chain of the form $\left[L_{-}, L_{+}\right]$, with $L_{-} \leqq 0<L_{+}$, and such that the number of sites is even.

For concreteness, let us suppose first that the number of sites $\left(L_{+}-L_{-}+1\right)$ is even. Then, the boundary strips are either both of the $A$ or both of the $B$ type; suppose they are of the $A$ type. Recall that we then have free boundary conditions for the $A$-clusters and wired boundary conditions for the $B$-clusters (wired independently at the left and at the right boundary) in the space (horizontal) direction. In the time (vertical) direction the boundary conditions are periodic. Call everything connected to the leftmost $B$-cluster the "outside." Then each loop in $\omega$ unambiguously encloses a domain and has the complement of that domain as its outside, 
i.e. every loop in $\omega$ is the outer boundary of exactly one domain and except for the "outside," each domain has a loop as its outer boundary.

Therefore the following relation holds:

$$
l_{\mathrm{per}}(\omega)=C_{A}(\omega)+C_{B}(\omega)-1 .
$$

Obviously we would have arrived at the same relation (3.7) if the boundary strips are of the $B$ type or if the number of sites is odd. Denote by $N_{A}(\omega)$ and $N_{B}(\omega)$ the number of activated bonds that occur in $\omega$ in the $A$-strips and $B$-strips, respectively. Then, with the boundary conditions described above, the following Euler relation holds (see Lemma 3.3 below):

$$
C_{B}(\omega)-C_{A}(\omega)+N_{A}(\omega)-N_{B}(\omega)=\frac{1}{2}\left\{(-1)^{L_{-}}-(-1)^{L_{+}}\right\} .
$$

Using (3.9) and (3.10) we have:

$$
\begin{aligned}
\rho^{J}(d \omega) u^{l_{\operatorname{pr}}(\omega)} & =\rho^{J}(d \omega) u^{C_{A}(\omega)+C_{B}(\omega)-1} \\
& =u^{\frac{1}{2}\left\{(-1)^{L-}-(-1)^{L+\}}-1\right.} \rho^{J}(d \omega) u^{2 C_{A}(\omega)-N_{A}(\omega)+N_{B}(\omega)} \\
& =u^{-\frac{1}{2}\left\{(-1)^{L-}-(-1)^{L+}\right\}-1} \rho^{J}(d \omega) u^{2 C_{B}(\omega)-N_{B}(\omega)+N_{A}(\omega)}
\end{aligned}
$$

The factors $u^{N_{A}(\omega)}$ and $u^{N_{B}(\omega)}$ can easily be absorbed in the measure $\rho(d \omega)$ as a mere modification of the intensity and the normalization of the Poisson process using the relation:

$$
\rho^{J}(d \omega) \lambda^{N(\omega)}=e^{\beta J(1-\lambda)} \rho^{\lambda J}(d \omega)
$$

for any $\lambda>0$. That completes the proof.

Proof of Theorem 3.1. In Proposition 2.1 we obtained the following expression for the partition function of the spin $S$ model with interaction $-P^{(0)}$ :

$$
\mathscr{Z}^{(S)}=\int \rho^{J}(d \omega)(2 S+1)^{l_{\operatorname{per}(\omega)}} .
$$

We apply the second equality of Proposition 3.2 with $u=2 S+1$. Comparing the result with (3.6) one then sees that up to a trivial constant $\mathscr{Z}^{(S)}$ is equal to the partition function of the $A$-Potts model in the Fortuin-Kasteleyn representation with $q=(2 S+1)^{2}$. Taking the logarithm one obtains i) of Theorem 3.1. In the same way one obtains ii).

We still have to prove the particular form of the Euler relation that was used in the proof of Proposition 3.2. We will use the following lemma also to determine the self-dual point of the "continuous time" Potts models.

Lemma 3.3 (The Euler relation). With the prescription of above one has:

$$
E(\omega) \equiv C_{B}(\omega)-C_{A}(\omega)+N_{A}(\omega)-N_{B}(\omega)=\frac{1}{2}\left\{(-1)^{L-}-(-1)^{L+}\right\} .
$$

Proof. It is obvious that (3.14) holds for the configuration $\omega$ that contains no bonds at all. We now prove that (3.14) is valid in general by showing that for any configuration $\omega^{\prime}$ obtained from any other configuration $\omega$ by removing a bond, one has $E\left(\omega^{\prime}\right)=E(\omega)$.

Suppose for concreteness that the bond to be removed is in an $A$-strip and call it $b$. We divide the neighborhood of $b$ into four regions and label them $A_{1}, A_{2}, B_{1}$ and 
$B_{2}$ as shown in Fig. 1. There are two possibilities:

1) In the cases where the domains $A_{1}$ and $A_{2}$ are connected with the bond $b$ present, we must have that after the bond has been removed $B_{1}$ and $B_{2}$ are no longer connected. So in this case removing $b$ leaves the number of $A$-clusters unchanged, but increases the number of $B$-clusters by 1 . Hence indeed $E\left(\omega^{\prime}\right)=E(\omega)$.

2) If with $b$ present $A_{1}$ and $A_{2}$ are not connected, then there at least one of these two $A$-clusters must be surrounded by a $B$-cluster that contains both $B_{1}$ and $B_{2}$. Therefore $B_{1}$ and $B_{2}$ will still be connected when $b$ is removed. So, in this case removing $b$ leaves the number of $B$-clusters unchanged and decreases the number of $A$-clusters by 1 . Again $E\left(\omega^{\prime}\right)=E(\omega)$.

The Potts models are exactly solvable at their self-dual point in the thermodynamic limit. For quantum spin chains this corresponds to the translation invariant case where $J_{x}=J$ for all $x$, and taking the limit $\beta \rightarrow \infty$. This relation follows from the next proposition.

Proposition 3.4. The relation

$$
\frac{J^{H}}{J^{V}}=q
$$

determines the self-dual point of the continuous time Potts model with partition function (3.2).

Proof. The statement is a direct consequence of the Euler relation. In Fig. 1 a certain configuration $\omega$ of cuts and bonds is depicted. The clusters consist of the pieces of vertical strips connected by the horizontal bonds in between. Recall that in the time direction the boundary conditions are periodic. In the horizontal direction the clusters at the boundary strips are subject to free boundary conditions. We also added an extra strip without bonds to the left and to the right of the interval. This is the situation where Lemma 3.3 holds and the $A$ and the $B$ Potts model are then exactly each others dual.

Using Lemma 3.3 and (3.12), the expression (3.6) for the partition function of the $A$ Potts model can then be transformed into the partition function of the $B$ Potts model with new coupling constants:

$$
\begin{aligned}
\mathscr{Z}_{A}^{\text {Potts }} & =\int \rho_{A}^{J^{V}}\left(d \omega_{A}\right) \rho_{B}^{J^{H}}\left(d \omega_{B}\right) q^{C_{A}\left(\omega_{A} \times \omega_{B}\right)} \\
& =q^{-\frac{1}{2}\left\{(-1)^{L-}-(-1)^{L}\right\}} \int \rho_{A}^{q J^{V}}\left(d \omega_{A}\right) \rho_{B}^{q^{-1} J^{H}}\left(d \omega_{B}\right) q^{C_{B}\left(\omega_{A} \times \omega_{B}\right)} .
\end{aligned}
$$

As $\rho_{A}^{q^{J V}}\left(\mathrm{~d} \omega_{A}\right)$ generates the horizontal and $\rho_{B}^{q^{-1} J^{H}}\left(d \omega_{B}\right)$ the vertical bonds of the $B$-model, the condition for self-duality is $J^{H}=q J^{V}$.

Note that because of the relations between the parameters of the quantum spin chain and the Potts models, self-duality of the Potts models is equivalent to translation invariance of the spin chain.

We end this section by deriving an expression for the spin-spin correlation function of the spin chain under consideration, in terms of the associated random cluster model (3.6). This random cluster model is a simultaneous realization of the $A$ Potts model and its dual, the $B$ Potts model. As remarked before, the boundary conditions are periodic in the vertical direction for both realizations, but at the left 
and right edges one has wired boundary conditions for one and free boundary conditions for the other realization.

In terms of the random loop model the (imaginary time) spin-chain correlation function is given by (see (2.9)):

$\left\langle S^{3}(x, t) S^{3}(y, s)\right\rangle=(-1)^{x-y} C(S) \operatorname{Prob}((x, t)$ is connected by a loop to $(y, s))$.

In the random cluster model the loops are viewed as the boundaries between two domains of opposite type. It is therefore clear that:

$$
\left\langle S^{3}(x, t) S^{3}(y, s)\right\rangle=(-1)^{x-y} C(S) \mu\left(I_{A}(\mathbf{x}, \mathbf{y}) I_{B}(\mathbf{x}, \mathbf{y})\right),
$$

where, for $C=A, B, I_{C}(\mathbf{x}, \mathbf{y})$ is the indicator function of the event that $\mathbf{x}_{C}$ and $\mathbf{y}_{C}$ belong to the same cluster. Here $\mathbf{x}_{C}=\left(x_{C}, t\right)$, with $x_{C}$ defined by the requirements that $\left|x-x_{C}\right|=\frac{1}{2}$, and that $x_{C}$ belongs to strip of type $C$. Note that for any two space-time points $\mathbf{x}$ and $\mathbf{y}, I_{A}(\mathbf{x}, \mathbf{y})$ is an increasing and $I_{B}(\mathbf{x}, \mathbf{y})$ a decreasing function.

We will often use the notation $\mathbf{x} \sim \mathbf{y}$ to indicate the event that $\mathbf{x}$ and $\mathbf{y}$ are connected. If $\mathbf{x}$ and $\mathbf{y}$ have integer space coordinates (i.e. they are on the vertical lines) connected means connected by a loop or a line in $\omega$. If the space coordinates of $\mathbf{x}$ and $\mathbf{y}$ are not integer the notation means that they belong to the same connected cluster. The event is empty if the two points belong to strips of different type or if the space coordinate of one of them is an integer and of the other one is not.

The individual probabilities $\operatorname{Prob}\left(\mathbf{x}_{A}\right.$ and $\mathbf{y}_{A}$ belong to the same cluster), can be computed in the $q$-state Potts model (see the proof of Proposition 3.2, where we applied the Euler relation). So,

$\left\langle S^{3}(\mathbf{x}) S^{3}(\mathbf{y})\right\rangle(-1)^{x-y} \leqq C(S) \operatorname{Prob}_{P_{\text {otts }}}\left(\mathbf{x}_{A}\right.$ and $\mathbf{y}_{A}$ belong to the same cluster) ,

and if $|x-y|=1$ and $t=s$, we have

$\left\langle S^{3}(x) S^{3}(x+1)\right\rangle=-\mathrm{C}(S) \operatorname{Prob}_{\text {Potts }}\left(x-\frac{1}{2}\right.$ and $x+\frac{3}{2}$ belong to the same cluster) .

\section{Finite Systems and the Thermodynamic Limit}

As a first application of the equivalence with the Potts model obtained in the previous section we now prove some preliminary properties of the spin $S$ model with interaction $-P^{(0)}$. Except when mentioned otherwise we only assume that the coupling constants are strictly positive.

Theorem 4.1 (Finite Systems). For each finite interval $\left[L_{-}, L_{+}\right]$, the limit

$$
\langle\cdot\rangle_{[L-, L+]}=\lim _{\beta \rightarrow \infty}\langle\cdot\rangle_{[L-, L+]}^{\beta}
$$

exists, both for the quantum spin chain and for the associated random cluster model. Furthermore, if the number of sites (i.e. $L_{+}-L_{-}+1$ ) is even, the limiting quantum state has $S_{\mathrm{tot}}=0$ and in the random cluster model there are no infinite lines (almost surely). 
That the finite volume ground state of a finite chain of even length is unique and is a spin singlet confirms the antiferromagnetic nature of the models. The above theorem can be viewed as the analogue of the well known result of Lieb and Mattis [42] for the Heisenberg antiferromagnet for the class of Hamiltonians under consideration here.

\section{Theorem 4.2 (The Thermodynamic Limit).}

1) The finite volume ground states for the quantum spin chains and the probability measures for the random cluster models converge to a well-defined thermodynamic limit provided the parity of the boundary sites is preserved; i.e. for any local observable $Q$ the following limits exist:

$$
\begin{gathered}
\langle Q\rangle_{\text {even }} \equiv \lim _{\substack{L-\rightarrow-\infty, L-\text { even } \\
L+\rightarrow+\infty, L+\infty \text { odd }}} \lim _{\beta \rightarrow \infty}\langle Q\rangle_{[L-, L+]}^{\beta}, \\
\langle Q\rangle_{\text {odd }} \equiv \lim _{\substack{L-\infty, L \text { odd } \\
L \rightarrow+\infty}} \lim _{\substack{L+\infty \\
\beta \rightarrow+\text { even }}}\langle Q\rangle_{[L-, L+]}^{\beta},
\end{gathered}
$$

and with similar limits defining $\mu_{\mathrm{even}}(d \omega)$ and $\mu_{\mathrm{odd}}(d \omega)$.

2) Therelation between the quantum states and the probability measure of the random cluster model valid in finite volume (see Sects. 2 and 3) persist in the infinite volume limit. In particular:

$\left\langle\mathbf{S}_{x} \cdot \mathbf{S}_{y}\right\rangle_{\text {even (odd) }}=(-1)^{x-y} C(S) \operatorname{Prob}_{\mu \text { even (odd) }}((x, 0)$ is connected by a loop to $(y, 0))$.

3) For translation invariant couplings the states $\langle\cdot\rangle_{\mathrm{even}}$ and $\langle\cdot\rangle_{\mathrm{odd}}$ are translates of each other, and each is invariant under translation of period two, as well as under global spin rotations.

Remark 4.3. For translation invariant and staggered couplings the states $\langle\cdot\rangle_{\text {even }}$ and $\langle\cdot\rangle_{\text {odd }}$ are also ergodic and weakly mixing [21] under even translations. This fact follows from the clustering relation and the clustering properties of the Potts model which are implied by FKG arguments (see Sect. 7 (Theorem 7.2)).

The rest of the section is devoted to the proofs of Theorems 4.1 and 4.2 .

Proof of Theorem 4.1. Since the arguments are fairly standard we shall be satisfied with an outline of the proof.

The existence of the limiting quantum state for the finite chains is trivial. For the random cluster model we can use the one-dimensionality of the system and the Perron-Frobenius theorem.

If the number of sites in the spin chain $\left(L_{+}-L+1\right)$ is even, it follows that if there is one infinite line, then there must be at least two infinite lines with opposite parity, i.e. such that at any given time the distance between the two lines is odd. In the random cluster picture this means that there are two infinite clusters of the same color $(A$ or $B)$ which is impossible by the theorem of Burton and Keane [12]. Alternatively, one can show by a variational argument that maintaining two infinite lines of opposite parity in a finite system costs a non-vanishing amount of free energy per unit of imaginary time.

By construction the state of the spin chain is necessarily rotation invariant and because there are no infinite lines one easily shows that $\left\langle f\left(S_{\text {tot }}^{3}\right)\right\rangle=f(0)$ for any function $f$. Hence the state must have $S_{\text {tot }}=0$. 
Proof of Theorem 4.2. From the FKG structure defined in Sect. 3 it follows that the measures $\mu_{\left[L_{-}, L_{+}\right]}(d \omega)$ form a monotone sequence in FKG sense (see Appendix II) if $L_{-}$and $L_{+}$are chosen such that the boundary strips are always of the same type $(A$ or $B)$. The standard argument then guarantees that the thermodynamic limit converges (see e.g. [1]).

That also for the quantum system the thermodynamic limit converges and satisfies the relation (4.4) is not immediately evident because local observables for the quantum chain are typically related to probabilities of a non-monotone and non-local event, e.g.

$$
\begin{aligned}
& \left\langle S_{x}^{3} S_{y}^{3}\right\rangle_{\text {even }} \\
& \quad=(-1)^{|x-y|} C(S) \lim _{\substack{L-\rightarrow-\infty, L-\text { even } \\
L+\rightarrow+\infty, L+\text { odd }}} \operatorname{Prob}_{\mu_{[L, L-3}}((x, 0) \text { is connected by a loop to }(y, 0)) .
\end{aligned}
$$

The event " $(x, 0)$ is connected by a loop to $(y, 0)$ " is non-local and it is nonmonotone because

$$
\begin{aligned}
& \operatorname{Prob}_{\text {loops }}(\mathbf{x} \text { and } \mathbf{y} \text { are connected by a loop) } \\
& \quad=\text { Prob }_{\text {random clusters }}\left(\begin{array}{l}
\mathbf{x}_{A} \text { and } \mathbf{y}_{A} \text { belong to the same cluster and } \\
\mathbf{x}_{B} \text { and } \mathbf{y}_{B} \text { belong to the same cluster }
\end{array}\right),
\end{aligned}
$$

is the probability of a non-monotone event since the connectivity of the $A$ clusters is increasing and the connectivity of the $B$ clusters is decreasing for the order structure at hand. Therefore we need a separate argument to show that

$$
\begin{gathered}
\lim _{\substack{L-\rightarrow-\infty, L-\text { even } \\
L+\rightarrow+\infty, L+\text { odd }}} \operatorname{Prob}_{\mu_{[L-L-]}}((x, 0) \text { is connected by a loop to }(y, 0)) \\
=\operatorname{Prob}_{\mu_{\text {even }}}((x, 0) \text { is connected by a loop to }(y, 0)) .
\end{gathered}
$$

If (4.7) holds (and also its obvious extensions to probabilities of more general connectivities of finite sets of sites), the result follows by Proposition 2.1.

For convenience we introduce the following shorthand notation:

$$
\begin{aligned}
L & \equiv\left[L_{-}, L_{+}\right], \\
\Lambda_{M} & \equiv[-M, M] \times[-M, M], \\
L \rightarrow \infty & \equiv \begin{array}{l}
L_{-} \rightarrow-\infty, L_{-} \text {even } \\
L_{+} \rightarrow+\infty, L_{+} \text {odd }
\end{array} \\
A_{L} & \equiv \operatorname{Prob}_{\mu_{[L-, L-]}}(x \text { is connected by a loop to } y), \\
A_{L, M} & \equiv \operatorname{Prob}_{\mu_{[L-, L-]}}\left(x \text { is connected to } y \text { in the box } \Lambda_{M}\right), \\
A_{\infty} & \equiv \operatorname{Prob}_{\mu_{\mathrm{even}}}(x \text { is connected by a loop to } y) .
\end{aligned}
$$

It is obvious that for each finite $L$,

$$
A_{L}=\sup _{M} A_{L, M},
$$


and also that

$$
0 \leqq A_{L}-A_{L, M} \leqq \operatorname{Prob}_{L}\left(\begin{array}{l}
x \text { and } y \text { are connected to } \Lambda_{M}^{c} \text { but not } \\
\text { to each other by a loop inside } \Lambda_{M}
\end{array}\right) .
$$

For $M$ fixed we take the $\lim \sup _{L \rightarrow \infty}$ of (4.10). For local events the lim sup is actually a convergent limit. Therefore:

$$
\begin{aligned}
& 0 \leqq \underset{L \rightarrow \infty}{\limsup } A_{L}-\operatorname{Prob}_{\mu_{\text {even }}}\left(x \text { and } y \text { are connected inside } \Lambda_{M}\right) \\
& \leqq \operatorname{Prob}_{\mu_{\text {even }}}\left(x \text { and } y \text { are connected to } \Lambda_{M}^{c} \text { but not inside } \Lambda_{M}\right) .
\end{aligned}
$$

The RHS is monotone decreasing in $M$. Taking the $\lim _{M \rightarrow \infty}$ :

$$
0 \leqq \underset{L \rightarrow \infty}{\limsup } A_{L}-A_{\infty} \leqq \operatorname{Prob}_{\mu_{\text {even }}}\left(x_{A} \text { and } y_{A} \text { belong to two distinct } \infty \text { clusters }\right)
$$

Since the RHS vanishes, the value of $\lim \sup _{L \rightarrow \infty} A_{L}$ is independent of the sequence of $L$ 's and so the limit exists and equals $A_{\infty}$.

\section{Absence of Néel Order}

An infinite volume ground state of a quantum spin chain is said to be Néel ordered if

$$
\liminf (-1)^{r}\left\langle S_{0} \cdot S_{r}\right\rangle>0 .
$$

The aim of this section is to prove that in the spin $S$ model with interaction $-P^{(0)}$ Néel order does not occur. More specifically we prove the following theorem.

Theorem 5.1 (Absence of Néel Order). For translation invariant or staggered coupling constants the infinite volume ground states $\langle\cdot\rangle_{\mathrm{even}}$ and $\langle\cdot\rangle_{\mathrm{odd}}$ satisfy:

$$
\lim _{r \rightarrow \infty}\left\langle\mathbf{S}_{0} \cdot \mathbf{S}_{\mathbf{r}}\right\rangle_{\substack{\text { even } \\ \text { (odd) }}}=0 .
$$

In absence of Néel order in the ground state of isotropic antiferromagnetic quantum spin chains is believed to hold quite generally, but a rigorous proof of this general fact is lacking. More was done for the Heisenberg antiferromagnetic chains: 1) for $S=1 / 2$ the exact Bethe Ansatz solution shows no Néel order, and 2) for general spin, an argument for the absence of Néel order was given in [48] on the basis of a new correlation inequality (which is further discussed in [49]).

A key role for the argument is played by a result of Gandolfi, Kean, and Russo [22], whose adaptation to our system takes the following form.

Proposition 5.2. For the wired state of the A Potts model

Prob(within a box of size $r,(0,0)$ is surrounded by an $A$-connected path) $\underset{r \rightarrow \infty}{\longrightarrow} 1$,

which holds regardless of $q$. 
It is easy to see that (5.3) is equivalent to the statement that there is no $B$-percolation in the state obtained with the boundary conditions which favor $A$-connections. The reason for that fact is that if the $A$-clusters percolate then, then any finite region is encircled by $A$-connected closed paths which prevent $B$ percolation. If there is no $A$-percolation in this $A$-preferred state then neither do $B$-clusters percolate. The proof of [22] rests on the planar nature of the connectivity graph (i.e. the nearest neighbor nature of the interaction), its reflection symmetries, and the FKG property.

Proof of Theorem 5.1. Theorem 4.2 permits us to express the expectation values of any local observable of the infinite spin chain in terms of the random cluster measures $\mu_{\text {even }}(d \omega)$ and $\mu_{\text {odd }}(d \omega)$. The correspondence is identical to what Proposition 2.1 provides for finite systems. In particular, by (2.9):

$$
(-1)^{r}\left\langle\mathbf{S}_{0} \cdot \mathbf{S}_{r}\right\rangle_{\text {odd }}=\operatorname{Prob}_{A-\text { wired }}((0,0) \text { and }(r, 0) \text { are on the same loop }) .
$$

As the loops are the boundaries of the connected clusters in the equivalent random cluster model, when two sites $\mathbf{x}$ and $\mathbf{y}$ are on the same loop then both $\mathbf{x}_{A} \sim \mathbf{y}_{A}$ (in the $A$-sense) and $\mathbf{x}_{B} \sim \mathbf{y}_{B}$ (in the $B$-sense). Hence the probability is bounded by

$\leqq 1-$ Prob(within a box of size $r$,

$(0,0)$ is surrounded by an $A$-connected path) $\underset{r \rightarrow \infty}{\longrightarrow} 0$,

where the last step is by Proposition 5.2.

Let us remark that assuming the validity of the exact results for the Potts model, absence of Néel order is also implied by the bound on the spin-spin correlation function in terms of the truncated two-point function of the Potts model, which is derived in Sect. 7.

\section{Dimerization versus Power Law Decay: A Dichotomy}

Despite the result of the last section, the models considered here may exhibit symmetry breaking. However, the symmetry is that of translation, and the phenomenon is caused by dimerization of the spin chain. The main result of this section is the following dichotomy: for the models considered here, the ground state either dimerizes (and exhibits spontaneous breaking of the translation invariance), or the spin-spin correlations have a slow (non-exponential) decay, satisfying

$$
\sum_{x}\left|x\left\langle S_{0}^{3} S_{x}^{3}\right\rangle\right|=+\infty
$$

Both possibilities occur (see the discussion at the end of Sect. 7).

A version of this dichotomy was first proved by Affleck and Lieb [7], extending a result of Lieb, Schulz and Mattis [43]. They prove that the uniqueness of the ground state implies the existence of low energy excitations (of the order of the inverse size of the system). The argument in [3] applies only to half-integer $\mathrm{SU}(2)$-spin chains, and to $\mathrm{SU}(n)$-spin chains with a self-conjugate representation 
acting at a site. Thus, the domains of applicability of our analysis and that of [7] have some partial overlap, but none includes the other.

We also show that, when translation invariance is spontaneously broken, the two periodic states are distinguished by the nearest neighbour spin-spin correlation function. In this sense (and more if one looks into the representation) the states are dimerized. The phenomenon can also be detected by the long distance behavior of quantity:

$$
\mathcal{O}_{x, y}=e^{i \frac{2 \pi}{2 S+1} \sum_{u=x}^{y} S_{u}^{3}},
$$

considered here only for $x<y \in \mathbb{Z}$ with $x-y$ odd.

Observables very similar to $\mathcal{O}_{x, y}$ have been used earlier in studies of ground states of quantum spin systems (see $[7,25,36,47]$ ) and in the computation of the magnetization of the critical Potts model [9]. In the second part of this section we introduce the "total spin on half of the infinite chain" as an operator in the GNS Hilbert space of the ground state. This quantity appears to us as of more fundamental significance than the string observables $\mathcal{O}_{x, y}$, and the latter can be expressed in terms of it. Moreover this new operator serves as a dimerization order parameter which reveals more clearly the detailed nature of the dimer order.

\subsection{The dichotomy. Following is the main result of this section.}

Theorem 6.1. For the ground states of the translation invariant spin $S$ model with interaction $-P^{(0)}\left((3.1)\right.$ with $\left.J_{x} \equiv J\right)$, the following dichotomy holds:

- either the translation symmetry is spontaneously broken in the infinite volume ground states

- or the spin-spin correlation function decays slowly (non-exponential) with

$$
\sum_{x}\left|x\left\langle S_{0}^{3} S_{x}^{3}\right\rangle\right|=+\infty
$$

In the first case, the symmetry breaking is manifested in the non-invariance of the pair correlation:

$$
\left\langle\mathbf{S}_{0} \cdot \mathbf{S}_{1}\right\rangle_{\text {even }} \neq\left\langle\mathbf{S}_{1} \cdot \mathbf{S}_{2}\right\rangle_{\text {even }}=\left\langle\mathbf{S}_{0} \cdot \mathbf{S}_{1}\right\rangle_{\text {odd }}
$$

and also in the string order parameter:

$$
\begin{aligned}
\lim _{N \rightarrow \infty}\left\langle\mathcal{O}_{0,2 N-1}\right\rangle_{\text {even }} & \left.=\lim _{N \rightarrow \infty}\left\langle\mathcal{O}_{1,2 N}\right\rangle_{\text {odd }}\right\rangle 0, \\
\lim _{N \rightarrow \infty}\left\langle\mathcal{O}_{1,2 N-1}\right\rangle_{\text {odd }} & =\lim _{N \rightarrow \infty}\left\langle\mathcal{O}_{1,2 N}\right\rangle_{\text {even }}=0 .
\end{aligned}
$$

Proof. We again rely on Theorem 4.2 to express the infinite volume state in terms of the random cluster measures $\mu_{\text {even }}(d \omega)$ and $\mu_{\text {odd }}(d \omega)$. Since the even and the odd states are translates of each other, it suffices to consider one of them, for concreteness say the odd state.

The physical origin of the dichotomy was outlined in the introduction. The random cluster representation permits us now to express these ideas in a precise way.

First we note that the result of [22], on the absence of simultaneous $A$ and $B$ percolation, implies that the configurations $\omega$ contain no infinite lines, i.e. the 
lines (in space-time) along which the spins are correlated occur only in the form of finite, non-intersecting, closed loops. The most important implication is that at any given time, the spins are locked into rigidly correlated even clusters, with $\sum S^{3}=0$ within each cluster.

The alternative may now be posed as between the following two possibilities: the number of loops surrounding each site is either almost surely finite, or it is almost surely infinite. Equivalently, there either is percolation or no percolation (and if there is percolation then either $A$ or $B$ percolates, never both, and hence the translation symmetry breaking). The zero-one nature of these probabilities is due to the ergodicity of the measures under even translations (Remark 4.3).

In the absence of percolation, by the identity (2.9):

$$
\begin{gathered}
\sum_{x \leqq 0, y \geqq 1}\left|\left\langle S_{x}^{3} S_{y}^{3}\right\rangle\right|=C(S) \sum_{x \leqq 0, y \geqq 1} \operatorname{Prob}((x, 0) \sim(y, 0)) \\
=C(S) \mathbb{E}_{\text {odd }}(\# \text { connected pairs }\{(x, 0),(y, 0)\}, \\
x \leqq 0, y \geqq 1)=\infty,
\end{gathered}
$$

where $\mathbb{E}_{\text {odd }}$ is the expectation with respect to the probability measure $\mu_{\text {odd }}$.

That the alternative necessitates symmetry breaking can be seen in different ways: i) via the existence of either $A$ or $B$ percolation, ii) by the distinction between the two sublattices in the values of a topological index, or iii) via the staggered values taken by the string order parameter. More explicitly:

i) If (6.1) fails then the origin is surrounded by a finite number of finite loops. The last of those necessarily touches the infinite cluster. For the random cluster state corresponding to the spin state $\langle\cdot\rangle_{\text {odd }}$, percolation is possible only for the $A$-cluster. (The argument is presented in Sect. 5). It then easily follows that

$$
\operatorname{Prob}_{\text {odd }}(x \text { belongs to an infinite cluster })=\left\{\begin{array}{ll}
m>0 & \text { if } x=\text { even }+\frac{1}{2} \\
0 & \text { if } x=\text { odd }+\frac{1}{2}
\end{array} .\right.
$$

A relevant question now is whether the probabilities of these events, which are expressed purely in terms of the configurations $\omega$, can be expressed in terms of the expectation values of some spin observables. As we show below, the answer is - yes!

ii) An alternative way to express the translation symmetry breaking, though still at the level of the random cluster measure, is by considering the topological index:

$$
w(\mathbf{x}, \omega)=(-1)^{\sum_{\gamma \in \omega} \mathbf{I}(\gamma \text { encloses } \mathbf{x})} .
$$

The index is defined only if the number of loops is finite, and it is easy to see that, when defined, its values alternate (with the overall phase dependent on $\omega$ ).

iii) We now show that the string order parameter (6.2), which is an observable of the quantum spin chain, directly detects cluster connectivity (as opposed to loop connectivity which determines the spin-spin correlation). By an application of $(2.6-7)$ :

$$
\left\langle\mathcal{O}_{x, y}\right\rangle_{\mathrm{even}(\mathrm{odd})}=\int \mu_{\mathrm{even} \mathrm{(odd)}} E_{\omega}\left(\mathcal{O}_{x, y}\right)
$$

with

$E_{\omega}\left(\mathcal{O}_{x, y}\right)=\mathbf{I}[$ each loop of $\omega$ intersects $[x, y]$ an even number of times] . 
We use here the observation that the net flux of any simple loop through an interval is either zero or \pm 1 . If $\omega$ has a loop with a non-zero flux through the interval $[x, y]$ then the conditional average of $\mathcal{O}_{x, y}$, over the consistent spin configurations, vanishes. In the other case, $\sum_{u=x}^{y} S_{u}^{3} \equiv 0$.

Hence, the string order parameter can be given a neat geometrical meaning:

$\left\langle\mathcal{O}_{x, y}\right\rangle=\operatorname{Prob}($ all loops intersect $[x, y]$ an even number of times)

$=$ Prob(any loop that encloses $\left(x-\frac{1}{2}, 0\right)$ also encloses $\left(y+\frac{1}{2}, 0\right)$ and vice versa)

$=\operatorname{Prob}\left(\left(x-\frac{1}{2}, 0\right)\right.$ and $\left(y+\frac{1}{2}, 0\right)$ belong to the same cluster $)$.

It immediately follows that $\left\langle\mathcal{O}_{x, y}\right\rangle=0$ if $y-x-1$ is odd.

For the odd boundary conditions the percolating cluster can only be of the $A$-type and it is unique. It follows that

$$
\begin{aligned}
\lim _{N \rightarrow \infty}\left\langle\mathcal{O}_{x, x+2 N+1}\right\rangle_{\text {odd }}= & \lim _{N \rightarrow \infty} \operatorname{Prob}_{\mu_{\text {odd }}}\left(\left(x-\frac{1}{2}, 0\right) \text { and }\left(x+2 N+\frac{3}{2}, 0\right)\right. \\
& \text { belong to the } \infty \text { cluster }) \\
= & \begin{cases}m^{2} & \text { for } x \text { odd } \\
0 & \text { for } x \text { even }\end{cases}
\end{aligned}
$$

Equation (6.13) is an explicit proof that the alternative to (6.7) is symmetry breaking.

Going beyond the last statement, we can also show that the translation symmetry breaking is necessarily manifested in the nearest neighbor correlations. By (4.4):

$$
\begin{aligned}
\left\langle S_{x}^{3} S_{x+1}^{3}\right\rangle_{\text {odd }} & =-C(S) \operatorname{Prob}_{\mu_{\text {odd }}}\left(x-\frac{1}{2} \sim x+\frac{3}{2}\right) \\
& = \begin{cases}\int \mu_{\text {odd }}(d \omega) I_{A}(x, x+1) & \text { if } x \text { is odd } \\
\int \mu_{\text {odd }}(d \omega) I_{B}(x, x+1) & \text { if } x \text { is even }\end{cases} \\
& = \begin{cases}\int \mu_{\text {odd }}(d \omega) I_{A}(x, x+1) & \text { if } x \text { is odd } \\
\int \mu_{\text {even }}(d \omega) I_{A}(x, x+1) & \text { if } x \text { is even }\end{cases}
\end{aligned}
$$

where we also used the duality relation.

When there is percolation, $A$-clusters percolate in the state $\mu_{\text {odd }}$ but not in the state $\mu_{\text {even }}$. The fact that this difference is then detected also at the level of the nearest-neighbor connections is an implication of the general criterion of the Rising Tide Lemma, which is derived here in Appendix II. (In the terminology explained there, $I_{C}(x, y)$ (with $\left.C=A, B\right)$ are strictly monotone functions.)

6.2. The dimerization order parameter. More can be said about the dimerized state in algebraic terms, by referring to the Hilbert space associated with the ground states $\langle\cdot\rangle_{\text {even }}$ and $\langle\cdot\rangle_{\text {odd }}$ via the GNS construction.

In physical terms, when it is correct to view the spins as organized into neutral clusters it is natural to talk about the total excess spin to the right of $x$, i.e., the total spin in half of the chain. This observation explains the following claim, which can be derived within our representation of the states $\langle\cdot\rangle_{\text {even }}$ and $\langle\cdot\rangle_{\text {odd }}$. 
Claim 6.2. Under the condition:

$$
\sum_{x}\left|x\left\langle S_{0}^{3} S_{x}^{3}\right\rangle\right|<\infty
$$

(i.e., the opposite of (6.1)) the following limits exist

$$
\widehat{S}_{x}^{3}=\lim _{\varepsilon \downarrow 0} \sum_{y>x} e^{-\varepsilon|y-x|} S_{y}^{3}=\lim _{\varepsilon \rightarrow 0} \hat{S}_{x}^{3}(\varepsilon),
$$

in the sense of strong resolvent convergence of operators in the GNS Hilbert space associated with either $\langle\cdot\rangle_{\text {even }}$ or $\langle\cdot\rangle_{\text {odd }}$.

In terms of the random cluster representation:

$$
\hat{S}_{x}^{3}=\sum_{y>x} S_{y}^{3} \mathbf{I}[\text { the loop of } y \text { intersects }(-\infty, x]] .
$$

We omit here the proof, except for the comment that what is proven explicitly is the convergence of the quantities:

$$
\left\langle A e^{i t \hat{S}_{x}^{3}(\varepsilon)} B\right\rangle_{\text {even (odd) }}
$$

for all strictly local spin observables. Equation (6.15) is a natural condition for both the existence of the limiting operator $\hat{S}_{x}^{3}$ and for the proof of the convergence. The first statement is the simpler task, e.g., it is easy to see that:

$$
\left\langle\left|\widehat{S}_{x}^{3}\right|^{2}\right\rangle_{\text {even (odd) }} \leqq \sum_{y>0}\left|y\left\langle S_{0}^{3} S_{y}^{3}\right\rangle\right|
$$

The operator $\hat{S}_{x}^{3}$ is the third component of a vector (under spin rotations), with $\widehat{S}_{x}^{1}$ and $\widehat{S}_{x}^{2}$ defined analogously. Using the invariance of the states:

$$
\left\langle e^{\frac{2 \pi i}{2 S+1} \hat{S}_{x}^{3}}\right\rangle_{\text {even (odd) }}=\left\langle P_{\left|\hat{S}_{x}\right|^{2}=0}\right\rangle_{\text {even (odd) }},
$$

where $P_{\left|\hat{S}_{x}\right|^{2}=0}$ is the orthogonal projection onto the subspace with $\left|\hat{S}_{x}\right|^{2}=0$.

The operators thus constructed permit to express the string quantity as follows:

$$
\mathcal{O}_{x, y}=e^{\frac{2 \pi i}{2 S+1}\left(\hat{S}_{x-1}^{3}-\hat{S}_{y}^{3}\right)} \text { for } y>x \in \mathbb{Z} .
$$

Under the condition (6.15), the formula (6.17) for $\hat{S}_{x}^{3}$, implies the clustering behavior:

$$
\left\langle\mathcal{O}_{x, y}\right\rangle_{\text {even (odd) }}-\left\langle P_{\left|\hat{S}_{x-1}\right|^{2}=0}\right\rangle\left\langle P_{\left|\hat{S}_{y}\right|^{2}=0}\right\rangle \rightarrow 0
$$

for $|x-y| \rightarrow \infty$. Hence, by comparison with (6.13) or directly from (6.17),

$$
\left\langle P_{\left|\hat{S}_{0}\right|^{2}=0}\right\rangle_{\text {odd }}=m, \quad\left\langle P_{\left|\hat{S}_{1}\right|^{2}=0}\right\rangle_{\text {odd }}=0 \text {. }
$$

Thus, the rotation of all the spins to the right of an odd site, by an angle $2 k \pi /(2 S+1), k=1, \ldots, 2 S$, produces a state orthogonal to the ground state $\langle\cdot\rangle_{\text {odd }}$. The orthogonality expresses the fact that such a rotation will necessarily break an existing bond. That is not the case for rotations of the spins to the right of an even site since, within the odd state, there is a positive probability that none of the clusters within which the spins are correlated are broken by this division.

Remark 6.3. In the above discussion we used percolation ideas to relate the failure of (6.1) to the positivity of $m$. For half integer spins, that can be replaced by an 
alternative argument based on the operators $\hat{S}_{x}^{3}$, which shows how remarkable is the fact of their existence. For half integer spins

$$
E_{\omega}\left(P_{\left|\hat{S}_{\lambda}\right| \in \mathbb{Z}}\right)=\mathbf{I}[w(x, \omega)=+1]=0,1,
$$

where the important observation is the $0-1$ property. Therefore, due to the ergodicity of the state $\mu_{\text {odd }}(d \omega)$ under the even translations, for each $x$ :

$$
\left\langle P_{\left|\hat{S}_{x}\right| \varepsilon \mathbb{Z}}\right\rangle_{\text {odd }}=0,1 \text { depending on the parity of } x \text {. }
$$

Since,

$$
\widehat{S}_{x}^{3}=\widehat{S}_{x+1}^{3}+S_{x+1}^{3}
$$

the projections obey

$$
P_{\left|\hat{S}_{x}\right| \in \mathbb{Z}}=1-P_{\left|\hat{S}_{x+1}\right| \in \mathbb{Z}} .
$$

That directly implies the lack of translation invariance, in the following explicit form:

$$
\begin{aligned}
\left\langle e^{2 \pi i \hat{S}_{x}^{3}}\right\rangle_{\text {odd }} & =\left\langle P_{\left|\hat{S}_{x}\right| \in \mathbb{Z}}\right\rangle_{\text {odd }}-\left\langle P_{\left|\hat{S}_{\mathrm{x}}\right| \in \mathbb{Z}+\frac{1}{2}}\right\rangle_{\text {odd }} \\
& =(-1)^{x},
\end{aligned}
$$

where the overall phase was determined by parity considerations.

This line of reasoning is reminiscent of the structural proof, by Aizenman and Martin [4], of symmetry breaking in one dimensional Coulomb systems. It may be noted that a string quantity related to the exponent seen in (6.28) was used in the argument of [7], which was also restricted to half integer spins.

The above argument expresses a different mechanism for dimerization than the one used in the proof of Theorem 6.1. The restriction to half-integer spin is compensated by the fact that (6.28) can be extended to a different class of (frustration free) Hamiltonians for which the percolation picture is not valid, where the result does hinge on the parity of $2 S$.

\section{Decay of Correlations in the Spin $S$ Model with Interaction $-P^{(0)}$}

Some elementary properties of the infinite volume limit of the states $\langle\cdot\rangle_{\text {even }}$ and $\langle\cdot\rangle_{\text {odd }}$ were discussed in Theorem 4.2. We now present sufficient conditions for the exponential decay of correlations in these states. The main result is:

Theorem 7.1. If either the A or the B Potts model associated with the ground state of the Hamiltonian

$$
H=-\sum_{x} J_{x} P_{x, x+1}^{(0)}, \quad J_{x}>0 \text { for all } x,
$$

has an exponentially decaying truncated pair correlation function (of the Potts variables), then the limiting states $\langle\cdot\rangle_{\mathrm{even}}$ and $\langle\cdot\rangle_{\mathrm{odd}}$ exhibit exponential decay of correlations of local observables, and a non-vanishing spectral gap above the ground state energy.

For a more explicit statement we introduce the following terminology. Let $Q$ be a local observable of the form

$$
Q=e^{-t H} \prod_{i=1}^{k} S_{x_{i}}^{\alpha_{t}} e^{t H}
$$


(any local observable can be written as a finite sum of such products). Then $\operatorname{supp} Q$ (the support of $Q$ ) is the set of space-time points $\left\{\left(x_{i}, t\right)\right\}$. We also define $\overline{\operatorname{supp}} Q$ to be the minimal interval (consisting of points at time $t$ with integer space coordinate) containing $\operatorname{supp} Q$.

The truncated correlation of two local observables $Q$ and $Q^{\prime}$, is defined as $\left\langle Q ; Q^{\prime}\right\rangle=\left\langle Q Q^{\prime}\right\rangle-\langle Q\rangle\left\langle Q^{\prime}\right\rangle$. For the $A$ Potts model the truncated two-point function, in a state which is symmetric under permutations of the $q$ values of the spin, has the random cluster interpretation

$$
\begin{aligned}
\tau_{A}(\mathbf{x}, \mathbf{y}) & =\left\langle\xi_{\mathbf{x}_{A}} \xi_{\mathbf{y}_{A}}\right\rangle-\left\langle\xi_{\mathbf{x}_{A}}\right\rangle\left\langle\xi_{\mathbf{y}_{A}}\right\rangle \\
& =\frac{c(q)}{q-1}\left(q\left\langle\delta_{\xi_{\mathbf{x}_{A}}, \xi_{\mathbf{y}_{A}}}\right\rangle-1\right) \\
& =c(q) \operatorname{Prob}\left(\mathbf{x}_{A} \text { and } \mathbf{y}_{A} \text { belong to the same connected cluster }\right),
\end{aligned}
$$

where

$$
c(q)=\frac{1}{12}\left(q^{2}-1\right)
$$

with a similar relation holding for the $B$ Potts model.

Theorem 7.2. Let $\langle\cdot\rangle$ denote the expectation in the ground state of a finite chain containing an even number of sites, or in one of the limiting states $\langle\cdot\rangle_{\text {even }}$ or $\langle\cdot\rangle_{\text {odd }}$ of Theorem 4.2. Then,for any pair of local observables of the quantum system of the form (7.2),

$$
\left|\left\langle Q ; Q^{\prime}\right\rangle\right| \leqq C_{Q} C_{Q^{\prime}} \sum_{\substack{y \in \operatorname{supp} \\ z \in \operatorname{supp}}} \underset{Q^{\prime}}{ } \min _{C=A, B} \tau_{C}(\mathbf{y}, \mathbf{z}),
$$

where $C_{Q}$ and $C_{Q^{\prime}}$ are invariant under space-time translations of the observables. The relations between the coupling constants of the quantum spin chain and the Potts models are given in Theorem 3.1.

The minimum over the two Potts models in (7.5) is important since it is expected that in any situation one of the correlation functions vanishes, as $(\mathbf{x}-\mathbf{y}) \rightarrow \infty$. If that is indeed the case, then the implication is that the limiting states $\langle\cdot\rangle_{\text {even }}$ and $\langle\cdot\rangle_{\text {odd }}$ are always clustering and hence pure phases. In the case of translation invariant or staggered couplings we have the following remark.

Remark 7.3. The following inequality is obvious:

$$
\tau_{C}(\mathbf{x}, \mathbf{y}) \leqq c(q) \operatorname{Prob}\left(\begin{array}{l}
\text { the connected cluster of } \mathbf{x}_{C} \text { reaches beyond } \\
\text { a box of size } 2\left\|\mathbf{x}_{C}-\mathbf{y}_{C}\right\| \text { centered at } \mathbf{x}_{C}
\end{array}\right) .
$$

Therefore, whenever one can show that there is no simultaneous percolation of the $A$-and $B$-clusters, one also obtains

$$
\lim _{\|\mathbf{x}-\mathbf{y}\| \rightarrow \infty} \min _{C=A, B} \tau_{C}(\mathbf{x}, \mathbf{y})=0
$$

In the case of translation invariant or staggered coupling constants the argument of [22] applies, but we expect that simultaneous $A$ and $B$ percolation is absent under much weaker conditions. 
The bound (7.5), and some of the arguments used in its derivation are reminiscent of the estimate derived in [2] for the quantum Ising model in transverse field. However, the case considered here is less direct. From (3.18) it is clear that the spin-spin correlation function of the quantum chain is not equal to the truncated two-point function of the associated Potts model. Nevertheless the decay rates of the two are equal (Theorem 7.6).

At the end of this section we discuss some implications for models with alternating coupling constants.

There are three steps to the proof of Theorem 7.2:

1) When the truncated correlations of quantum spins are transcribed in terms of the Potts model, we obtain two distinct contributions. The first is easily bounded in terms of the random cluster model's connectivity function, and the second is a truncated correlation function of suitable observables of the random cluster model.

2) Using a general domination principle for FKG measures, the latter correlation function is bound in terms of four point functions of the form

$$
\left\langle I_{C_{1}}(\mathbf{x}, \mathbf{y}) ; I_{C_{2}}(\mathbf{u}, \mathbf{v})\right\rangle,
$$

where $C_{i}$ are either $A$ or $B$, and $I_{C}(\mathbf{x}, \mathbf{y})$ are indicator functions for the events that $\mathbf{x}_{C}$ and $\mathbf{y}_{C}$ are in the same connected $C$-cluster (for the definition of $\mathbf{x}_{C}$ see at the end of Sect. 3).

3) It is shown that for any combination of $C_{1}$ and $C_{2}$ the truncated correlation (7.8) is bounded in terms of the connectivity function of the $A$-model. By symmetry, the same is true for the $B$-model (in any situation, only one of these bounds will have a non-trivial consequence). The argument is based on a combination of the FKG inequality and the Markov property of the random cluster measure.

The argument relies on two auxiliary results. The first one, used in step 2 , is the following domination principle for correlation functions with respect to an FKG measure.

Lemma 7.4 ([46]). For a pair of functions $f$ and $g$, let $F$ and $G$ be two monotone functions with which $f+F$ and $g+G$ are increasing and $f-F$ and $g-G$ are decreasing. Then the truncated correlations with respect to any measure with the FKG property satisfy:

$$
|\langle f ; g\rangle| \leqq\langle F ; G\rangle \text {. }
$$

The proof is elementary: it consists of two linear combinations of the four correlation inequalities resulting from the monotonicity of $f \pm F$ and $g \pm G$.

The next Lemma is needed for step 3. It provides an upper bound for the truncated correlation of two monotone increasing functions of the random cluster model.

Lemma 7.5. Let $I_{1}$ and $I_{2}$ be two monotone increasing events for the random cluster model, which are determined by the A-connected clusters of two non-random sets $D_{1}$ and $D_{2}$. Then:

$$
0 \leqq\left\langle I_{1} ; I_{2}\right\rangle \leqq \operatorname{Prob}\left(D_{1} \text { and } D_{2} \text { are } A \text {-connected }\right) .
$$

Proof. The key observation is the following inequality which results from the combination of the Markov structure and the FKG property of the random cluster measure:

$$
\mathbb{E}\left[I_{2} \mid I\left[D_{1} \nsucc D_{2}\right] I_{1}\right] \leqq\left\langle I_{2}\right\rangle
$$


The reason for this inequality is that in the complement of $C_{A}\left[D_{1}\right]$, the $A$ connected cluster of $D_{1}$, the system can be considered as having free boundary conditions on the boundary. These boundary conditions mask the positive event $I_{1}$ which is determined within $C_{A}\left[D_{1}\right]$.

The above reasoning was first employed in [13] for the Ising model and similar arguments have later been used for a variety of other applications.

Using (7.11) and the trivial bound $\left|I_{1}\right| \leqq 1$, one then obtains:

$$
\begin{aligned}
\frac{\left\langle I_{1} I_{2}\right\rangle}{\left\langle I_{2}\right\rangle}=\mathbb{E}\left[I_{1} \mid I_{2}\right]= & \mathbb{E}\left[I_{1} \mid I\left[D_{1} \sim D_{2}\right] I_{2}\right] \mathbb{E}\left[I\left[D_{1} \sim D_{2}\right] \mid I_{2}\right] \\
& +\mathbb{E}\left[I_{1} \mid I\left[D_{1} \nsucc D_{2}\right] I_{2}\right] \mathbb{E}\left[I\left[D_{1} \sim D_{2}\right] \mid I_{2}\right] \\
\leqq & \mathbb{E}\left[I\left[D_{1} \sim D_{2}\right] \mid I_{2}\right]+\left\langle I_{1}\right\rangle .
\end{aligned}
$$

Thus,

$$
\left\langle I_{1}\right\rangle\left\langle I_{2}\right\rangle \leqq\left\langle I_{1} I_{2}\right\rangle \leqq\left\langle I_{1}\right\rangle\left\langle I_{2}\right\rangle+\mathbb{E}\left[I\left[D_{1} \sim D_{2}\right]\right],
$$

where the first inequality is just by FKG. That implies the bound stated in the Lemma for the truncated correlation $\left\langle I_{1} ; I_{2}\right\rangle$.

Proof of Theorem 7.2. For convenience, we first carry the analysis for observables which are products, of the form (7.2), of only $S^{1}$ and $S^{3}$ variables at non-coincidental sites, and break the proof into the steps described above.

1) In terms of the random cluster representation of the spin chains:

$$
\left\langle Q ; Q^{\prime}\right\rangle=\int \mu(d \omega) E_{\omega}\left(Q Q^{\prime}\right)-\left(\int \mu(d \omega) E_{\omega}(Q)\right)\left(\int \mu\left(d \omega^{\prime}\right) E_{\omega^{\prime}}\left(Q^{\prime}\right)\right) .
$$

Therefore

$$
\begin{aligned}
\left.K Q ; Q^{\prime}\right\rangle \mid \leqq & \left|\int \mu(d \omega) E_{\omega}\left(Q Q^{\prime}\right)-\int \mu(d \omega) E_{\omega}(Q) E_{\omega}\left(Q^{\prime}\right)\right| \\
& +\left|\int \mu(d \omega) E_{\omega}(Q) E_{\omega}\left(Q^{\prime}\right)-\left(\int \mu(d \omega) E_{\omega}(Q)\right)\left(\int \mu\left(d \omega^{\prime}\right) E_{\omega^{\prime}}\left(Q^{\prime}\right)\right)\right| .
\end{aligned}
$$

The first term on the right side can be interpreted as the average over $\omega$ of the truncated correlation within the quasi states $E_{\omega}$. The second term is a truncated correlation function of the random cluster model.

To eliminate the first term we note the factorization property (see (2.37))

$$
\begin{array}{ll}
E_{\omega}\left(Q Q^{\prime}\right)=E_{\omega}(Q) E_{\omega}\left(Q^{\prime}\right) & \text { if } \omega \text { does not contain a loop } \\
& \text { connecting } \operatorname{supp} Q \text { and } \operatorname{supp} Q^{\prime} .
\end{array}
$$

Using also the bound $\left|E_{\omega}(Q)\right| \leqq S^{|\operatorname{supp} Q|}$, one has

$$
\begin{aligned}
& \left|\int \mu(d \omega) E_{\omega}\left(Q Q^{\prime}\right)-\int \mu(d \omega) E_{\omega}(Q) E_{\omega}\left(Q^{\prime}\right)\right| \\
& \quad \leqq S^{|\operatorname{supp} Q|+\left|\operatorname{supp} Q^{\prime}\right|} \sum_{\substack{\mathbf{x} \in \operatorname{supp} Q \\
\mathbf{y} \in \sup Q^{\prime}}} \operatorname{Prob}(\mathbf{x} \text { and } \mathbf{y} \text { are connected by a loop) } .
\end{aligned}
$$


When $\mathbf{x}$ and $\mathbf{y}$ are connected by a loop then both $\mathbf{x}_{A} \sim \mathbf{y}_{A}$ and $\mathbf{x}_{B} \sim \mathbf{y}_{B}$, where $\mathbf{x}_{C} \sim \mathbf{y}_{C}$ is our notation for the event that $\mathbf{x}_{C}$ and $\mathbf{y}_{C}$ belong to the same $C$ cluster. Thus we can continue the bound as:

$$
\begin{aligned}
& \leqq S^{|\operatorname{supp} Q|+\left|\operatorname{supp} Q^{\prime}\right|} \sum_{\substack{\mathbf{x} \in \operatorname{supp} Q \\
\mathbf{y} \in \operatorname{supp} Q^{\prime}}} \min _{C=A, B} \operatorname{Prob}\left(\mathbf{x}_{C} \sim \mathbf{y}_{C}\right) \\
& \leqq c(q)^{-1} S^{|\operatorname{supp} Q|+\left|\operatorname{supp} Q^{\prime}\right|} \sum_{\substack{\mathbf{y} \in \operatorname{supp} Q \\
\mathbf{z} \in \operatorname{supp} Q^{\prime}}} \min _{C=A, B} \tau_{C}(\mathbf{y}, \mathbf{z}) .
\end{aligned}
$$

2) In order to estimate the second term in the RHS of (7.15) we invoke Lemma 7.4. For the functions

$$
f(\omega)=E_{\omega}(Q), \quad g(\omega)=E_{\omega}\left(Q^{\prime}\right)
$$

we take

and

$$
F(\omega)=2 S^{|\operatorname{supp} Q|}\left(\sum_{\mathbf{x}, \mathbf{y} \in \operatorname{supp} Q} I_{A}(\mathbf{x}, \mathbf{y})-I_{B}(\mathbf{x}, \mathbf{y})\right)
$$

$$
G(\omega)=2 S^{\left|\operatorname{supp} Q^{\prime}\right\rangle}\left(\sum_{\mathbf{x}, \mathbf{y} \in \operatorname{supp} Q^{\prime}} I_{A}(\mathbf{x}, \mathbf{y})-I_{B}(\mathbf{x}, \mathbf{y})\right) .
$$

That the conditions of Lemma 7.4 are satisfied follows from the following observations. An elementary change of a configuration $\omega$ consists of the addition or the removal of a single bond. Such a change will either join a pair of loops or cut a loop into two. If $E_{\omega}(Q)$ is affected, the change is by not more than $2 S^{|\operatorname{supp} Q|}$. However, the change is zero unless there is a pair of sites $\mathbf{x}, \mathbf{y} \in \operatorname{supp} Q$ whose loops are either joined or disconnected in the process. In this situation the value of the increasing function $\sum_{\mathbf{x}, \mathbf{y} \in \operatorname{supp} Q} I_{A}(\mathbf{x}, \mathbf{y})-I_{B}(\mathbf{x}, \mathbf{y})$ is changed by at least 1 .

Applying the Lemma we get:

$$
\begin{aligned}
& \left|\int \mu(d \omega) E_{\omega}(Q) E_{\omega}\left(Q^{\prime}\right)-\left(\int \mu(d \omega) E_{\omega}(Q)\right)\left(\int \mu(d \omega) E_{\omega}\left(Q^{\prime}\right)\right)\right| \\
& \leqq 4 S^{|\operatorname{supp} Q|+\left|\operatorname{supp} Q^{\prime}\right|} \sum_{C_{1}, C_{2}} \sum_{\substack{\mathbf{x}, \mathbf{y} \in \operatorname{supp} Q \\
\mathbf{u}, \mathbf{v} \in \operatorname{supp} Q^{\prime}}}\left|\left\langle I_{C_{1}}(\mathbf{x}, \mathbf{y}) ; I_{C_{2}}(\mathbf{u}, \mathbf{v})\right\rangle\right|
\end{aligned}
$$

which concludes the second step of the proof.

3) Our goal now is to estimate the quantities $\left\langle I_{C_{1}}(\mathbf{x}, \mathbf{y}) ; I_{C_{2}}(\mathbf{u}, \mathbf{v})\right\rangle$ appearing in the right side of (7.22). By the " $A-B$ " symmetry, it suffices to derive an estimate in terms of the $A$-model's connectivity function $\tau_{A}(\cdot, \cdot)$.

The events $I_{C}(\mathbf{x}, \mathbf{y})$ are of two possible types:

i) two $A$-sites, $\mathbf{x}_{A}$ and $\mathbf{y}_{A}$, are in the same connected $A$-cluster, or

ii) two $B$-sites, $\mathbf{x}_{B}$ and $\mathbf{y}_{B}$, are not separated by a connected $A$-cluster (i.e. are connected by a $B$-cluster).

In either case, the events are determined by the $A$ cluster of the set $\left[\mathbf{x}_{C}, \mathbf{y}_{C}\right]_{A}$ defined by:

$\left[\mathbf{x}_{A}, \mathbf{y}_{A}\right]_{A}=$ the two point set $\left\{\mathbf{x}_{A}, \mathbf{y}_{A}\right\}$,

$\left[\mathbf{x}_{B}, \mathbf{y}_{B}\right]_{A}=$ the collection of the $A$ sites in the interval joining $\mathbf{x}_{B}$ and $\mathbf{y}_{B}$. 
For the application of Lemma 7.5 we define two monotone increasing events as follows: if $C_{1}=A$ put $I_{1}=I_{A}(\mathbf{x}, \mathbf{y})$ and $D_{1}=\left[\mathbf{x}_{A}, \mathbf{y}_{A}\right]_{A}$; if $C_{1}=B$ put $I_{1}=1-I_{B}(\mathbf{x}, \mathbf{y})$ and $D_{1}=\left[\mathbf{x}_{B}, \mathbf{y}_{B}\right]_{A} . I_{2}$ and $D_{2}$ are defined in terms of $I_{C_{2}}(\mathbf{u}, \mathbf{v})$ and $\left[\mathbf{u}_{C_{2}}, \mathbf{v}_{C_{2}}\right]_{A}$ in the same way. Lemma 7.5 then implies:

$$
\begin{aligned}
\left|\left\langle I_{C_{1}}(\mathbf{x}, \mathbf{y}) ; I_{C_{2}}(\mathbf{u}, \mathbf{v})\right\rangle\right| & \leqq \operatorname{Prob}\left(\left[\mathbf{x}_{C_{1}}, \mathbf{y}_{C_{1}}\right]_{A} \sim\left[\mathbf{u}_{C_{2}}, \mathbf{v}_{\mathbf{C}_{2}}\right]_{A}\right) \\
& \leqq \sum_{\substack{\mathbf{z} \in\left[\mathbf{x}_{1}, \mathbf{u}_{\mathbf{c}_{1}}\right]_{A} \\
\mathbf{z}^{\prime} \in\left[\mathbf{u}_{C_{2}}, \mathbf{v}_{C_{2}}\right]_{A}}} \operatorname{Prob}\left(\mathbf{z}_{A} \sim \mathbf{z}_{A}^{\prime}\right),
\end{aligned}
$$

where we have also used that $\left|\left\langle 1-I_{1} ; I_{2}\right\rangle\right|=\left|\left\langle I_{1} ; I_{2}\right\rangle\right|$.

Interchanging the rôles of $A$ and $B$ and using (7.3) one finally obtains the estimate

$$
\left|\left\langle I_{C_{1}}(\mathbf{x}, \mathbf{y}) ; I_{C_{2}}(\mathbf{u}, \mathbf{v})\right\rangle\right| \leqq c(q)^{-1} \min _{C=A, B} \sum_{\substack{\mathbf{z} \in\left[\mathbf{x}_{1}, \mathbf{y}_{c_{1}}\right] c \\ \mathbf{z}^{\prime} \in\left[\mathbf{u}_{c_{2},}, \mathbf{v}_{c_{2}}\right] c}} \tau_{C}\left(\mathbf{z}, \mathbf{z}^{\prime}\right) .
$$

Combining the inequalities (7.15), (7.18), (7.22), and (7.26), one obtains the estimate (7.5), for the case where $Q$ and $Q^{\prime}$ are products of $S^{1}$ and $S^{3}$ operators at distinct sites.

For the general case we use the identity $S^{2}=i\left[S^{1}, S^{3}\right]$ to express the product of spin operators as a linear combination of products of only $S^{1}$ and $S^{3}$. The products may contain repeated factors. With a trivial modification the argument given above applies to such products as well.

Proof of Theorem 7.1. Theorem 7.1 is a direct consequence of Theorem 7.2.

It is interesting to consider the implications of the above analysis for the spin chains with alternating coupling constants

$$
J_{x}=\left\{\begin{array}{ll}
J_{\text {even }} & \text { if } x \text { is even } \\
J_{\text {odd }} & \text { if } x \text { is odd }
\end{array} .\right.
$$

First it should be appreciated that while the spin system's Hamiltonian is only periodic, the associated Potts models are translation invariant. A great deal is known about such Potts models (though at varying levels of mathematical rigor), and our methods allow one to extract from that some relevant information on the ground states of the quantum spin chains.

For the Potts models it is known that the correlation length is a meaningful notion, defined by the limit

$$
\xi_{\text {Potts }}^{-1}=-\lim _{x \rightarrow \infty} \frac{1}{|x|} \ln \tau_{C}(0, x) .
$$

The existence of the limit is an easy consequence of the supermultiplicativity (itself implied by an FKG type argument): $\tau_{0}(x, z) \geqq \tau_{0}(x, y) \tau_{0}(y, z)$, where $\tau_{0}=c(q)^{-1} \tau_{C}$, $C=A$ or $B$.

Since Theorem 7.2 provides only upper bounds, let us first strengthen the relationship between the long distance behavior of the correlations in the two models.

Theorem 7.6. For the models with alternating coupling constants (7.27): if $\xi_{\text {Potts }}<\infty$ then also

exists and $\xi_{\text {Potts }}=\xi_{\mathrm{QSChain}}$.

$$
\xi_{\mathrm{QSChain}}^{-1}=-\lim _{x \rightarrow \infty} \frac{1}{|x|} \ln \left|\left\langle S_{0}^{3} S_{x}^{3}\right\rangle\right|
$$


Proof. Let $0<m=\xi_{\text {Potts }}^{-1}$ be defined by the limit (7.28). The theorem will follow from the following inequalities: for any $\varepsilon>0$ not too large, there exist constants $C_{+}, C_{-}>0$ such that

$$
C_{+} e^{-m(1-\varepsilon)|x|} \geqq\left|\left\langle S_{0}^{3}, S_{x}^{3}\right\rangle\right| \geqq C_{-} e^{-m(1+\varepsilon)|x|}
$$

for all $x$ large enough. Since the spin-spin correlations are dominated by the function $\tau(\cdot)$, the upper bound is trivial (in fact, an auxiliary argument shows that $C(S) e^{-m|x|}$ would do). We now provide the argument for the lower bound on the spin-spin correlation function. For concreteness let us assume that there is no percolation in the $A$ Potts model.

By (2.8) the problem amounts to estimating from below

$$
P(0, x) \equiv \operatorname{Prob}(0 \text { and } x \text { are on the same loop) . }
$$

We first show that for all small $\varepsilon>0$ and finite $D>0(D$ will be taken $\mathbf{O}(1))$ the following quantity satisfies an exponential lower bound as in (7.30):

$P_{\varepsilon, D}(0, x) \equiv \operatorname{Prob}\left(\begin{array}{l}\text { there are sites } u \in\left[(-4 \varepsilon x)_{A}, 0\right], v \in\left[x,(x+4 \varepsilon x)_{A}\right], \\ \text { such that } u \text { and } v \text { are on the same loop and each of } \\ \text { the } A \text {-strips at the edges of the two intervals does not } \\ \text { contain any bonds at times } t \in[-D, D]\end{array}\right)$

with $x$ odd. As the outer boundary of any $A$-cluster is a loop for the spin chain we obviously have:

$P_{\varepsilon, D}(0, x) \geqq$

Prob $\left(\begin{array}{l}\text { there is an } A \text {-cluster intersecting both }[-4 \varepsilon x, 0] \text { and }[x, x+ \\ 4 \varepsilon x] \text { but not }[-4 \varepsilon x, x+4 \varepsilon x]^{c} \text {, and each of the } A \text {-strips } \\ \text { centered at }(-4 \varepsilon x)_{A}, \frac{1}{2}, x-\frac{1}{2} \text {, and }(x+4 \varepsilon x)_{A} \text { does not } \\ \text { contain any bonds at times } t \in[-D, D]\end{array}\right)$,

and therefore

$P_{\varepsilon, D}(0, x) \geqq$

Prob $\left(\begin{array}{l}\exists \text { an } A \text {-cluster connecting }[-\infty, 0] \text { and }[x,+\infty] \text { and each of } \\ \text { the } A \text {-strips centered at }(-4 \varepsilon x)_{A}, \frac{1}{2}, x-\frac{1}{2} \text {, and }(x+4 \varepsilon x)_{A} \\ \text { does not contain any bonds at times } t \in[-D, D]\end{array}\right)$,

- Prob $(\exists$ an $A$-cluster connecting $[-\infty, 0]$ and $[x+4 \varepsilon x,+\infty])$

$-\operatorname{Prob}(\exists$ an $A$-cluster connecting $[-\infty,-4 \varepsilon x]$ and $[x,+\infty])$.

i) By FKG and translation invariance

$\operatorname{Prob}\left(\begin{array}{l}\exists \text { an } A \text {-cluster connecting }[-\infty, 0] \text { and }[x,+\infty] \text { and each of } \\ \text { the } A \text {-strips centered at }(-4 \varepsilon x)_{A}, \frac{1}{2}, x-\frac{1}{2} \text {, and }(x+4 \varepsilon x)_{A} \\ \text { does not contain any bonds at times } t \in[-D, D]\end{array}\right)$ 
$\geqq \operatorname{Prob}(\exists$ an $A$-cluster connecting $[-\infty, 0]$ and $[x+\infty])$

$\times$ Prob(the $A$-strip centered at $\frac{1}{2}$ does not contain any bonds at times $t \in[-D, D])^{4}$

and, using (7.28),

$$
\begin{gathered}
\text { Prob }[\exists A \text {-cluster connecting }[-\infty, 0] \text { and } \\
[x,+\infty]) \geqq c(q)^{-1} \tau_{A}(0, x) \geqq C^{\prime} e^{-(1+\varepsilon) m|x|} .
\end{gathered}
$$

ii) The middle term in (7.32) can be replaced by the straightforward estimate $\operatorname{Prob}(\exists A$-cluster intersecting both $[-\infty, 0]$ and $[x+4 \varepsilon x,+\infty])$

$$
\begin{aligned}
& \leqq \sum_{y \leqq 0, z \geqq x} \tau_{A}(y, z) \\
& \leqq C^{\prime \prime} e^{-(1+2 \varepsilon) m|x|},
\end{aligned}
$$

where $C^{\prime \prime}>0$ depends only on $m$, and it is assumed that $0<\varepsilon<\frac{1}{4}$. By symmetry, (7.34) applies also to the last term in (7.32). Combining (7.32-33) we obtain the desired bound for $P_{\varepsilon, D}(0, x)$ : for some $C>0$ (depending on $\left.D\right)$ :

$$
P_{\varepsilon, D}(0, x) \geqq C\left(1-C e^{-\varepsilon m|x|}\right) e^{-(1+\varepsilon) m|x|} .
$$

In order to complete the proof we have to argue that the exponential lower bound on $P_{\varepsilon, D}(0, x)$ implies a similar lower bound on $P(0, x)$. More precisely, we use the bound for $P_{\varepsilon, D}(0, x)$, which we only need for "0" even and $x$ odd, to obtain the desired estimate on $P(y, z)$ with $y=-1$ or $y=0$ and $z=x$ or $z=x+1$. This covers all possible combinations of parities for the two sites.

We use Lemma 7.7 given below to show that there is some $\alpha>0$ such that for $y=-1$ or 0 and $z=x$ or $x+1$ :

$\operatorname{Prob}(y$ and $z$ are on the same loop $\mid$ the event described in $(7.31)) \geqq e^{-m \alpha \varepsilon|x|}$,

from which the desired bound immediately follows.

The basis for the claim (7.35) are the following three observations:

i) The event described in (7.31) implies the existence of a line (forming part of a loop) outside of the rectangular neighborhoods $B_{1} \equiv\left[(-4 \varepsilon x)_{A}, 0\right] \times[-D, D]$ and $B_{2} \equiv\left[x,(x+4 \varepsilon x)_{A}\right] \times[-D, D]$, connecting a point of the form $(u, s)$ with a point $(v, t)$, where $u \in\left[(-4 \varepsilon x)_{A}, 0\right], v \in\left[x,(x+4 \varepsilon x)_{A}\right]$ and $s, t= \pm D$.

ii) For every pair of points $(u, s)$ and $(v, t)$, on the boundary of the boxes $B_{1}$ and $B_{2}$ respectively (see i)), there is a pair of local events $\mathscr{E}_{1}$ and $\mathscr{E}_{2}$ in the boxes $B_{1}$ and $B_{2}$ such that under $\mathscr{E}_{1}(u, s)$ is connected by a line to $(-1,0)$ or $(0,0)$, ad libitum, and under $\mathscr{E}_{2}(v, t)$ is connected with $(x, 0)$ or $(x+1,0)$. The events, whose choice depends on $u, s, v$, and $t$, are depicted in Fig. 3 .

iii) The conditional probability of the above mentioned local events $\mathscr{E}_{1}$ and $\mathscr{E}_{2}$ occurring together - conditioned on any explicit configuration in the complement of $B_{1}$ and $B_{2}$-is not smaller than $e^{-m \alpha \varepsilon|x|}$, for some $\alpha>0$.

More explicitly, the local events are constructed as follows (see Fig. 3). Assuming the line connecting $B_{1}$ and $B_{2}$ (in the complement of $B_{1}$ and $B_{2}$ ), reaches $B_{1}$ at the point $\mathbf{u}_{0}(u, D)$. The event $\mathscr{E}_{1}\left(\mathbf{u}_{0}\right)$ inside $B_{1}$ is then described by specifying that in 


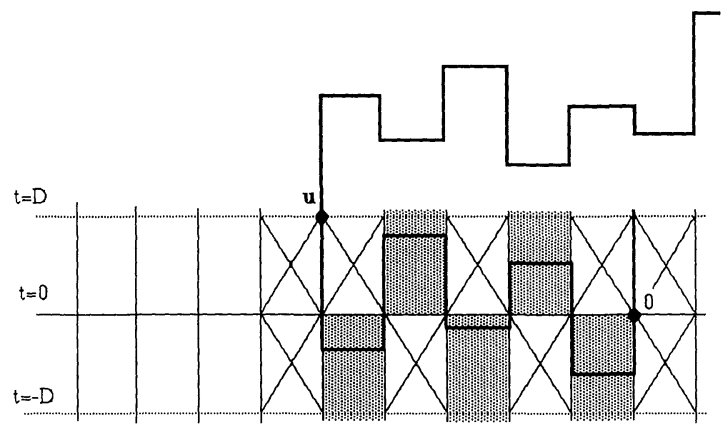

Fig. 3. A schematic representation of the local event $\mathscr{E}_{1}$ used in the proof of Theorem 7.6. Crossed areas do not contain any bonds and gray areas contain at least one bond

the vertical strips between $u$ and 0 one sees alternatingly the following picture: in the first, third, fifth, ... strip, counted starting from $u$, there is no bond at times $0 \leqq t \leqq D$ and at least one bond at a time $-D \leqq t \leqq 0$; in the second, fourth, ... strip there is at least one bond with $0 \leqq t \leqq D$ and no bond with $-D \leqq t \leqq 0$. The strip to the left of $u$ is required not to contain any bonds at times $-D \leqq t \leqq D$. It is then obvious that in the box $B_{1}$ there will be a line connecting $(u, D)$ and $(-1,0)$ and $(0,0)$. The case $u=0$ is treated by a trivial modification of the above prescription. The event $\mathscr{E}_{2}\left(\mathbf{v}_{0}\right)$ in $B_{2}$ is defined in a similar way.

Note that the random loop measure $\mu$ conditioned on an arbitrary configuration outside any finite domain in space-time is of the form stated in Lemma 7.7. The function $f$ is the number of loops inside the finite volume taking into account the connections in the configurations outside, and $q=2 S+1$. It is then obvious that $f$ always satisfies the bounded-gradient condition with $a=b=1$ because the addition or removal of a single bond can change the number of loops by at most one.

The indicator function of the event $\mathscr{E}_{1} \cap \mathscr{E}_{2}$ is of the form $F G$ with $F$ increasing and $G$ decreasing.

It is useful to introduce the auxiliary events $\mathscr{E}_{\mathbf{u}_{0}, \mathbf{v}_{0}}$, which form a partition of the event described in (7.31): $\left\{\mathbf{u}_{0}=(u, s), \mathbf{v}_{0}=(v, t)\right\}$ is the "first" pair of points on the top or bottom boundaries of $B_{1}$ and $B_{2}$ connected by a line outside these boxes ("first" e.g. in lexicographic order). We also define $J_{\min (\max )}=\min (\max )\left\{J_{\text {even }}, J_{\text {odd }}\right\}$ and put $D=1$.

The observations i-iii) and Lemma 7.7 then yield for $y=(-1,0)$ or $(0,0)$ and $z=(x, 0)$ or $(x+1,0)$ :

$\operatorname{Prob}(y$ and $z$ are on the same loop | the event described in (7.31))

$$
\begin{aligned}
& \geqq \sum_{\substack{\mathbf{u}_{0} \in \partial B_{1} \\
\mathbf{v}_{0} \in \partial B_{2}}} \operatorname{Prob}\left(\mathscr{E}_{1}\left(\mathbf{u}_{0}\right) \cap \mathscr{E}_{2}\left(\mathbf{v}_{0}\right) \mid \mathscr{E}_{\mathbf{u}_{0}, \mathbf{v}_{0}}\right) \operatorname{Prob}\left(\mathscr{E}_{\mathbf{u}_{0}, \mathbf{v}_{0}} \mid \text { the event described in }(7.31)\right) \\
& \geqq\left\{\int \rho^{J_{\min } /(2 S+1)}(d \omega) \mathbf{I}[\text { there is at least one bond in the strip }[0,1] \times[0,1]]\right\}^{2[\varepsilon|x|]} \\
& \quad \times\left\{\int \rho^{(2 S+1) J_{\max }}(d \omega) \mathbf{I}[\text { there is no bond in the strip }[0,1] \times[0,1]]\right\}^{2[\varepsilon|x|]}
\end{aligned}
$$




$$
\begin{aligned}
& \times\left\{\int \rho^{(2 S+1) J_{\max }}(d \omega) \mathbf{I}[\text { there is no bond in the strip }[0,1] \times[-1,1]]\right\}^{2} \\
= & e^{-4(2 S+1) J_{\max }}\left\{\left(1-e^{-J_{\min } /(2 S+1)}\right) e^{-(2 S+1) J_{\max }}\right\}^{2[\varepsilon|x|]} \\
\geqq & C e^{-m \alpha \varepsilon|x|}
\end{aligned}
$$

for some $\alpha>0$ independent of $\varepsilon$ and $x$. We can now conclude that for $\varepsilon>0$ small enough one has

$$
P(0, x) \geqq e^{-m \alpha \varepsilon|x|} P_{\varepsilon}(0, x) \geqq e^{-m(1+(\alpha+1) \varepsilon)|x|}
$$

for all $x$ large enough, which is a lower bound of the form (7.30).

In the above argument we use the following lemma, in which we refer to the order structure on the space of configurations given by the inclusion relation: $\omega \geqq \omega^{\prime}$ iff the set of bonds in $\omega$ contains the set of bonds in $\omega^{\prime}$. The proof is a rather standard FKG-type argument, and is therefore omitted here.

Lemma 7.7. Let $\mu(d \omega)$ be a probability measure of the form:

$$
\mu(d \omega)=\frac{\rho_{\Lambda}^{J}(d \omega) q^{f(\omega)}}{\int \rho_{\Lambda}^{J}(d \omega) q^{f(\omega)}},
$$

where $\rho_{\Lambda}^{J}(d \omega)$ is a Poisson measure on configurations $\omega$ in a finite volume $\Lambda$ (in our case, $\Lambda$ is a subset of the space-time), $q \geqq 1$, and $f(\omega)$ is a function of bounded gradient, in the sense that there are constants, $a, b \geqq 0$ such that $f+a N$ is non-decreasing and $f-b N$ is non-increasing with $N(\omega)$ the total number of time-indexed bonds in $\omega$. Then, for any two functions, $F$ non-decreasing and $G$ non-increasing, which depend on $\omega$ in two disjoint subsets of $\Lambda$ (one determining $F$ and the other determining $G$ ) one has the following comparison inequalities, with expectation values with respect to modified Poisson measures:

$$
\begin{aligned}
& \int \mu(d \omega) F(\omega) G(\omega) \leqq \int \rho_{\Lambda}^{q^{b} J}(d \omega) F(\omega) \int \rho_{\Lambda}^{q^{-a} J}(d \omega) G(\omega), \\
& \int \mu(d \omega) F(\omega) G(\omega) \geqq \int \rho_{\Lambda}^{q^{-a} J}(d \omega) F(\omega) \int \rho_{\Lambda}^{q^{b} J}(d \omega) G(\omega) .
\end{aligned}
$$

Assuming now the validity of all the results on Potts models presented in references $[31,40,41,54]$ (not all of which have been derived rigorously), we obtain the following implications.

i) If $J_{\text {even }} \neq J_{\text {odd }}$, the ground state is unique, with exponential decay of correlations and a spectral gap.

A perturbative version of this statement, for small (or large) enough ratio of the two couplings, is contained in a theorem of Kennedy and Tasaki [36].

ii) The case $J_{\text {even }}=J_{\text {odd }}$ corresponds to self dual Potts models, in which case there is a dichotomy, which we have discussed above in Sect. 6. Its manifestation in the Potts model language is: at the self dual point there either is a first order phase transition with coexistence of the ordered and disordered phases, or the transition is second order - with a unique state at which the correlations decay by a power law. The threshold value of $q$ is $q=4$, which corresponds to $S=1 / 2$. The implication of the Potts model calculation is:

ii.a For $S=1 / 2$, there is a unique state, but with algebraic decay of correlations and no spectral gap (Eq. (6.3) is satisfied). For the spin system that was known independently of the Potts model result, by the Bethe Ansatz solution and a result of [43]. 
ii.b For $S>1 / 2$ the translation invariance is spontaneously broken and there are two partially dimerized ground states, translates of each other, with exponential decay of correlations and a non-vanishing spectral gap (the exact value of the spectral gap can be calculated, see $[9,38,39]$ ).

iii) In the case $S=\frac{1}{2}$, for weakly staggered coupling constants, $J_{\text {even }}=1+\delta$, $J_{\text {odd }}=1-\delta, 0<\delta \ll 1$, the correlation length is finite but a divergent function of $\delta$. The conjectured values of the corresponding exponents of the 4-state Potts model [54], yield the following behaviour for the ground state energy per site $\varepsilon$ and the correlation length $\xi$ :

$$
\varepsilon-\varepsilon_{0} \sim-|\delta|^{\frac{4}{3}}, \quad \xi \sim|\delta|^{-\frac{2}{3}}
$$

(up to logarithmic corrections).

This behavior of the spin $\frac{1}{2}$ Heisenberg antiferromagnetic chain with alternating coupling strengths was first obtained by Cross and Fisher in their study of the spin-Peierls transition [15]. It has the implication that when the elastic deformations of the underlying lattice are taken into account, the ground state of spin$\frac{1}{2}$ Heisenberg antiferromagnetic chain develops the spin-Peierls instability. (For a related rigorous result, see e.g. [34]).

\section{Appendix I. Quasi-State Decomposition for Quantum States}

Often, basic properties of the state of a quantum system are elucidated by presenting it as a convex combination of states with a particularly simple structure. In this work we find it useful to consider a broader class of affine decompositions - into convex combinations of what is called below quasi-states. These are linear functionals which are required to meet the positivity requirements (which are part of the definition of a quantum state) only in their restrictions to certain Abelian subalgebras.

Quasi-state decompositions made already an implicit appearance in the discussion of the itinerant ferromagnetism in reference [3]. The utility of such decompositions there, stems from the fact that the ferromagnetic (or antiferromagnetic) properties of a system with rotation-invariant spin-spin couplings can be expressed through the correlation functions of a commuting family of the spin variables (e.g., $\left\{\sigma_{x}^{3}\right\}$ with $x$ the site index and 3 referring to the third component of the Pauli spin matrices). The structure of the restriction of the state to such families is made particularly transparent by decomposing it into a convex combination of states in which the correlations are either " 0 or 1 ," in the sense that spins are either locked in a parallel (or antiparallel) state, or are completely independent. This is also the major characteristic of the Fortuin-Kasteleyn representation of the Ising model $[19,18]$.

The purpose of this section is to formulate this notion, and discuss some of its general properties in the context of a simple example.

\section{I.a Definition of Quasi-States}

Definition 1.1. Let $\mathscr{A}$ be a sub-algebra of observables of a quantum system. A densely defined linear functional $\rho$ (providing the expectation value for observables) is called 
a quasi-state relative to $\mathscr{A}$, and we will say that it is well adapted to $\mathscr{A}$, if $\rho$ is:

i) normalized: $\rho(\mathbb{1})=1$,

ii) positive on $\mathscr{A}$, i.e. for each bounded $A \in \mathscr{A}, \rho\left(A^{*} A\right) \geqq 0$.

The property of states which is not required of quasi-states is the general positivity, i.e., the unrestricted validity of (I.2).

For finite dimensional quantum systems, the observables form matrix algebras, and the linear functionals take the form:

$$
\rho(A)=\operatorname{Tr} Q A,
$$

where $Q$ is a corresponding density matrix. When $\mathscr{A}$ is the maximal-Abelian algebra of matrices which are diagonal in a certain common basis $\left\{\left|\alpha_{n}\right\rangle\right\}$, the conditions on $Q$ under which $\rho$ is a quasi-state relative to $\mathscr{A}$ are: i) $\operatorname{Tr} Q=1$, and ii) $Q$ has non-negative diagonal elements,

$$
\left\langle\alpha_{n}|Q| \alpha_{n}\right\rangle \geqq 0 \text { for } n=1, \ldots .
$$

The last requirement is considerably weaker than the condition $\mathrm{ii}^{\prime}$ ) $Q \geqq 0$ (as a matrix) needed for $\rho$ to be a state.

Remark I.2. At this point it is natural to ask whether (I.4) has any $\mathscr{A}$-independent content. The answer is rather negative: any hermitian matrix with $\operatorname{Tr} Q \geqq 0$ satisfies (I.4) in some basis.

As we shall see, in certain situations a convex decomposition of a state into quasi-states (relative to a naturally relevant algebra), provides a great deal of insight into a state's structure. Such decompositions take the form:

$$
\rho=\sum_{n} p_{n} Q_{n}
$$

with the $Q_{n}$ quasi-states relative to a common algebra, and $p_{n}$ weights satisfying:

$$
p_{n} \geqq 0, \quad \sum_{n} p_{n}=1
$$

I.b. An Elementary Example. As the simplest demonstration of the notion introduced above, consider the system consisting of two spin-1/2 objects, with the spin-spin ferromagnetic interaction:

$$
H=-\vec{\sigma}_{1} \cdot \vec{\sigma}_{2} .
$$

The ground state of the related antiferromagnetic Hamiltonian $(-H)$ is given by the rank-one projection

$$
P_{0}=|0\rangle\langle 0|
$$

onto the single state $\left(|0\rangle=\left(\left|\frac{1}{2},-\frac{1}{2}\right\rangle-\left|-\frac{1}{2}, \frac{1}{2}\right\rangle\right) / \sqrt{2}\right)$, where the total spin is $S=0$. In this state one can safely say that $\vec{\sigma}_{1}=-\vec{\sigma}_{2}$, e.g.,

$$
\operatorname{Tr} P_{0}\left(\vec{\sigma}_{1}+\vec{\sigma}_{2}\right)^{2}=0 \text {. }
$$

The ground state of the ferromagnet is slightly less elementary. It has the threefold degeneracy of the space on which $S=1$. The corresponding projection is 
$P_{1}=\mathbb{1}-P_{0}=S(\mathrm{~S}$ takes here only the values 0 and 1$)$, and the (normalized) state operator is

$$
Q_{+}=\frac{1}{3} S .
$$

While the spins are as parallel as the uncertainty relations allow, it is not true that $\vec{\sigma}_{1}-\vec{\sigma}_{2}=0$. In fact,

$$
\operatorname{Tr} Q_{+}\left(\vec{\sigma}_{1}-\vec{\sigma}_{2}\right)^{2}=1>0 .
$$

The fact that except for the "zero-point fluctuations" the spins are basically aligned, is easily seen in the following quasi-state decomposition of the ferromagnetic ground state:

$$
Q_{+}=\frac{2}{3}\left(\frac{1}{4}\right) \mathbb{1}+\frac{1}{3}\left(\frac{1}{2} T\right),
$$

where $T=2 S-1$ is the exchange operator $(T|a, b\rangle=|b, a\rangle ; a, b= \pm)$.

Two basic observations here are:

- For any non-zero vector $\mathbf{n} \in \mathbb{R}^{3}$ both $\frac{1}{4} \mathbb{1}$ and $\frac{1}{2} T$ are quasi-state operators relative to the Abelian algebra $\mathscr{A}_{\mathbf{n}}$ generated by $\left\{\overrightarrow{\boldsymbol{\sigma}}_{1} \cdot \mathbf{n}, \overrightarrow{\boldsymbol{\sigma}}_{2} \cdot \mathbf{n}\right\}$. The former is actually a state operator.

- On any of the Abelian factors $\mathscr{A}_{\mathrm{n}}$, the quasi-states corresponding to $\frac{1}{4} \mathbb{1}$ and to $\frac{1}{2} T$ represent two simple alternatives. In the first state, $\overrightarrow{\boldsymbol{\sigma}}_{1} \cdot \mathbf{n}$ and $\overrightarrow{\boldsymbol{\sigma}}_{2} \cdot \mathbf{n}$ form a pair of uncorrelated variables (taking the values $\pm \frac{1}{2}$ independently of each other), whereas in the quasi-state $\frac{1}{2} T, \overrightarrow{\boldsymbol{\sigma}}_{1} \cdot \mathbf{n}$ and $\overrightarrow{\boldsymbol{\sigma}}_{2} \cdot \mathbf{n}$ are locked together.

By the rotational symmetry, it suffices to derive these statements for $\mathbf{n}=(0,0,1)$, within the standard Pauli spin matrix representation, which makes the first statement really obvious. For the second we note that the restriction of $\operatorname{Tr}\left(\frac{1}{2} T\right) A$ to $A \in \mathscr{A}_{(0,0,1)}$ is determined by only the diagonal elements of $\frac{1}{2} T$ in the basis which diagonalizes $\left\{\sigma_{1}^{3}, \sigma_{2}^{3}\right\}$. That diagonal part of $\frac{1}{2} T$ is $\frac{1}{2}\left(\left|\frac{1}{2}, \frac{1}{2}\right\rangle\left\langle\frac{1}{2}, \frac{1}{2}|+|-\frac{1}{2},-\frac{1}{2}\right\rangle\left\langle-\frac{1}{2}\right.\right.$, $-\frac{1}{2} \mid$ ). In particular, it follows that the quasi-state $\frac{1}{2} T$ yields the following ferromagnetic analog of (I.9):

$$
\operatorname{Tr} \frac{1}{2} T\left(\overrightarrow{\boldsymbol{\sigma}}_{1}-\overrightarrow{\boldsymbol{\sigma}}_{2}\right)^{2}=0 .
$$

Thus, insofar as the restriction to $\mathscr{A}_{\mathbf{n}}$ is concerned, the ground state of the quantum ferromagnetic Hamiltonian is equivalent to a positive temperature state of a classical ferromagnet, and the decomposition (I.12) is identical to the Fortuin-Kasteleyn random cluster decomposition of the latter.

I.c. Words of caution. Some notes of caution are due here. The lack of full positivity implies that quasi-states may lack some of the properties which are familiar for the expectation value functionals $\langle\cdot\rangle$ associated with regular states, such as:

i) $A \geqq 0 \Rightarrow\langle A\rangle \geqq 0$,

ii) $\langle A\rangle \leqq\|A\|$ (the supremum norm of $A$ ),

iii) $\left\langle A^{*} A\right\rangle=0 \Rightarrow\langle A B\rangle=0$ for any (bounded) $B$.

In fact, the quasi-state operator $\frac{1}{2} T$ provides us with the following "counterexamples":

i) $\operatorname{Tr} \frac{1}{2} T\left[\left(\overrightarrow{\boldsymbol{\sigma}}_{1}-\overrightarrow{\boldsymbol{\sigma}}_{2}\right)^{2}-1\right]=-1$, while $\left(\overrightarrow{\boldsymbol{\sigma}}_{1}-\overrightarrow{\boldsymbol{\sigma}}_{2}\right)^{2}-1 \geqq 0$,

ii) $\operatorname{Tr} \frac{1}{2} T\left[2-\left(\overrightarrow{\boldsymbol{\sigma}}_{1}-\overrightarrow{\boldsymbol{\sigma}}_{2}\right)^{2}\right]=2$, while $\left\|2-\left(\overrightarrow{\boldsymbol{\sigma}}_{1}-\overrightarrow{\boldsymbol{\sigma}}_{2}\right)^{2}\right\|=1$, 
and

iii) $\operatorname{Tr} \frac{1}{2} T\left(\sigma_{1}^{3}-\sigma_{2}^{3}\right)^{2}=0$, yet $\operatorname{Tr} \frac{1}{2} T\left(\sigma_{1}^{3}-\sigma_{2}^{3}\right)\left\{T\left|-\frac{1}{2}, \frac{1}{2}\right\rangle\left\langle-\frac{1}{2}, \frac{1}{2}\right|\right\}[=1] \neq 0$.

These elementary assertions follow from (I.13) and the observation that $2-\left(\overrightarrow{\boldsymbol{\sigma}}_{1}-\overrightarrow{\boldsymbol{\sigma}}_{2}\right)^{2}=2 \mathrm{~S}-1= \pm 1$.

Nevertheless, the restriction of a quasi-state to any of the algebras for which it is well adapted is free of the above "pathologies." In particular, the three principles (I.14)-(I.16) are satisfied as long as $A$ and $B$ are restricted to any common $\mathscr{A}_{\mathbf{n}}$.

One may also note that while a quasi-state is not fully characterized by its restriction to a single Abelian factor, in the above case the operator $T$ is uniquely determined by its rotation invariance and the values of $\operatorname{Tr} \frac{1}{2} T A$ for $A \in \mathscr{A}_{\mathrm{n}}$.

\section{Appendix II: Strictly Monotone Observables for Gibbs-Fields and the Rising Tide Lemma}

The FKG structure of the probability measures $\mu_{\text {even }}(d \omega)$ and $\mu_{\text {odd }}(d \omega)$ is quite essential for our analysis in Sects. 4-7. Here we provide some details concerning this structure. We also introduce the notion of weak strict monotonicity and prove the Rising Tide Lemma which was needed in Theorem 6.1 to show that breaking of translation invariance $\left(\mu_{\text {even }} \neq \mu_{\text {odd }}\right)$ implies staggered behavior of the nearest neighbor spin-spin correlation $\left(\left\langle\mathbf{S}_{x} \cdot \mathbf{S}_{x+1}\right\rangle_{\text {even (odd) }} \neq\left\langle\mathbf{S}_{x+1} \cdot \mathbf{S}_{x+2}\right\rangle_{\text {even (odd) }}\right)$.

Recall from Sect. 3 the definition of the partial order on the set of configurations $\omega=\left(\omega_{A}, \omega_{B}\right)$, with $\omega_{A(B)}$ the set of $A(B)$-bonds labeled by space-time points. The partial order is defined by declaring

$$
\omega \preceq \omega^{\prime} \quad \text { if } \omega_{A} \supset \omega_{A}^{\prime} \text { and } \omega_{B} \subset \omega_{B}^{\prime} .
$$

That leads to the notions of monotonicity of functions and domination of measures: i) A function $f$ is called monotone increasing if

$$
f(\omega) \leqq f\left(\omega^{\prime}\right) \text { for all } \omega \preceq \omega^{\prime} .
$$

ii) A probability measure $\mu(d \omega)$ is said to be dominated by another probability measure $v(d \omega)$, denoted as $\mu(d \omega) \preceq v(d \omega)$, if

$$
\int \mu(d \omega) f(\omega) \leqq \int v(d \omega) f(\omega) \text { for all monotone increasing } f \text {. }
$$

This structure is useful in a number of ways.

1) Fortuin, Kasteleyn and Ginibre [20] provide a general criterion, under which a probability measure would have the property:

$$
\int \mu(d \omega) f(\omega) g(\omega) \geqq \int \mu(d \omega) f(\omega) \int \mu(d \omega) g(\omega)
$$

for all monotone increasing $f$ and $g$. The $\mathrm{FKG}$ condition is satisfied by the random cluster measures (see [1]).

2) For $\mu$ satisfying (II.4), the probability measures conditioned on the configuration in the complement of a finite volume, are monotone in the sense:

$$
\mathbb{E}_{\mu}\left(\cdot \mid \omega_{\Lambda^{c}}\right) \preceq \mathbb{E}_{\mu}\left(\cdot \mid \omega_{\Lambda^{c}}^{\prime}\right) \text { for all } \omega_{\Lambda^{c}} \preceq \omega_{\Lambda^{c}}^{\prime} .
$$

3) There exist domination relations among the random cluster measures corresponding to Potts models with various values of $q(q=1$ corresponding to 
independent percolation). Applications of these relations can be found in $[19,20$, $18]$ and [1].

4) Of particular interest in this work is the domination relation

$$
\mu_{\text {even }}(d \omega) \preceq \mu_{\text {odd }}(d \omega),
$$

which follows by a simple limiting argument from (II.5).

The last relation includes the statement $\left\langle\mathbf{S}_{0} \cdot \mathbf{S}_{1}\right\rangle_{\text {odd }} \leqq\left\langle\mathbf{S}_{0} \cdot \mathbf{S}_{1}\right\rangle_{\text {even }}$. Our goal now is to explain why whenever the two states are different, the inequality holds in the strict sense. A key role in the argument is played by Lemma II.2, whose name draws on the observation that a rising tide lifts all the ships in the harbor.

It is useful to introduce the notion of strict monotonicity.

Definition II.1. Let $\mathscr{M}$ be a set of probability measures on a configuration space $\Omega$ with an order structure as above. A monotone increasing function on $\Omega$ is strictly increasing in the weak sense with respect to $\mathscr{M}$ (for short $\mathscr{M}$-strictly increasing), if

$$
\int \mu(d \omega) f(\omega)<\int v(d \omega) f(\omega) \text { for all } \mu, v \in \mathscr{M}, \mu \preceq v \text { and } \mu \neq v .
$$

The difference between the two random cluster measures is due only to the boundary conditions (pushed to infinity). They share, however, a common rule for the finite volume conditional distributions. (A phenomenon exhibited also by the family of the Gibbs equilibrium states at a first order phase transition.) In the terminology discussed in [23] the measures have the same set of specifications.

Lemma II.2 (The Rising Tide Lemma). Let $\mathscr{M}$ be a family of probability measures with common specifications $\gamma=\left\{\mathbb{E}\left(\cdot \mid \omega_{\Lambda^{c}}\right)\right\}_{\Lambda}$. A sufficient condition for a function $f$ to be $\mathscr{M}$-strictly monotone increasing is that beyond some finite volume $\Lambda_{0}$ the conditional expectation is strictly monotone increasing with respect to the boundary conditions:

$$
\mathbb{E}\left(f \mid \omega_{\Lambda^{c}}\right)<\mathbb{E}\left(f \mid \omega_{\Lambda^{\prime}}\right)
$$

for all $\Lambda \supset \Lambda_{0}$ and all pairs of boundary conditions such that

$$
\omega_{\Lambda^{c}} \leq \omega_{\Lambda^{c}}^{\prime} \text { and } \omega_{\Lambda^{c}} \neq \omega_{\Lambda^{c}}^{\prime} \bmod \gamma,
$$

where the last inequality means that the induced conditional expectations are not identical.

Remark II.3. For a given set of specifications, the relevant notion of boundary conditions consists of the equivalence classes of $\left\{\omega_{\Lambda^{c}}\right\}$ defined by

$$
\omega_{\Lambda^{c}} \cong \omega_{\Lambda^{c}}^{\prime} \quad \text { iff } \mathbb{E}\left(\cdot \mid \omega_{\Lambda^{c}}\right)=\mathbb{E}\left(\cdot \mid \omega_{\Lambda^{c}}^{\prime}\right) .
$$

It is easy to see that for the random cluster model the equivalence class of boundary configurations is determined by specifying the connectivity relations of the boundary sites via the connecting paths in $\Lambda^{c}$. (Obviously many configurations in the complement of $\Lambda$ would be equivalent.)

Proof of the Lemma. Let $\mu$ and $v \in \mathscr{M}, \mu \preceq v$. Then, by the general result of Holley ([32]), there is a coupling $\rho$ which is a probability measure on the space of pairs $\left\{\left(\omega, \omega^{\prime}\right)\right\}$ with marginals $\mu$ and $v$ which is supported on pairs with $\omega \preceq \omega^{\prime}$. Using 
first the conditional expectation formula and then the coupling, we have:

$$
\begin{aligned}
v(f)-\mu(f) & =\int v\left(d \omega_{\Lambda^{c}}\right) \mathbb{E}\left(f \mid \omega_{\Lambda^{c}}\right)-\int \mu\left(d \omega_{\Lambda^{c}}^{\prime}\right) \mathbb{E}\left(f \mid \omega_{\Lambda^{c}}^{\prime}\right) \\
& =\int \rho\left(d \omega_{\Lambda^{c}} \times d \omega_{\Lambda^{c}}^{\prime}\right)\left(\mathbb{E}\left(f \mid \omega_{\Lambda^{c}}\right)-\mathbb{E}\left(f \mid \omega_{\Lambda^{c}}^{\prime}\right)\right) .
\end{aligned}
$$

Assuming the measures are different, there is some finite volume $\Lambda \supset \Lambda_{0}$ for which $\rho$ assigns a positive measure to pairs of configurations which are inequivalent as boundary conditions in $\Lambda^{c}$. Since the integrand in (II.11) is strictly positive at such points, and is non-negative in general, it follows that $v(f)-\mu(f)>0$.

Lemma II.3. For the random cluster model, for any $\mathbf{x}, \mathbf{y} \in \mathbb{Z} \times \mathbb{R}$, the random variable $I_{A}(\mathbf{x}, \mathbf{y})$ satisfy the criterion of the Rising Tide Lemma II.2. (The set $\Lambda_{0}$ can be taken as any box with $\mathbf{x}$ and $\mathbf{y}$ in its interior.)

Proof. Let $\Lambda$ be a finite box containing $\mathbf{x}$ and $\mathbf{y}$ in its interior, and let $\omega_{\Lambda^{c}} \preceq \omega_{\Lambda^{c}}^{\prime}$. Then each of these configurations induces a partition of the $A$-sites on the boundary of $\Lambda$ into clusters connected in the exterior. The relation between the configurations means that the boundary partition corresponding to $\omega_{\Lambda^{c}}$ is a refinement of that corresponding to $\omega_{\Lambda^{c}}^{\prime}$. The difference in the conditional expectations of $I_{A}(\mathbf{x}, \mathbf{y})$ is the result of two effects: i) the induced measures in $\Lambda$ are different, and ii) the finite volume conditions under which $x$ and $y$ are connected are different (since $\omega^{\prime}$ provides a better connected boundary). Denoting by $\mathbf{I}_{\omega_{\Lambda}{ }^{c}}$ and $\mathbf{I}_{\omega^{\prime} \Lambda^{c}}$ the corresponding indicator functions (of only $\omega_{\Lambda}$ ), we have:

$$
\begin{aligned}
\mathbb{E} & \left(I_{A}(\mathbf{x}, \mathbf{y}) \mid \omega_{\Lambda^{c}}^{\prime}\right)-\mathbb{E}\left(I_{A}(\mathbf{x}, \mathbf{y}) \mid \omega_{\Lambda^{c}}\right) \\
& =\mathbb{E}\left(\mathbf{I}_{\omega^{\prime} \Lambda^{c}}(\mathbf{x}, \mathbf{y}) \mid \omega_{\Lambda^{c}}^{\prime}\right)-\mathbb{E}\left(\mathbf{I}_{\omega_{\Lambda^{c}}}(\mathbf{x}, \mathbf{y}) \mid \omega_{\Lambda^{c}}\right) \\
& =\mathbb{E}\left(\mathbf{I}_{\omega_{\Lambda^{c}}}(\mathbf{x}, \mathbf{y}) \mid \omega_{\Lambda^{c}}^{\prime}\right)-\mathbb{E}\left(\mathbf{I}_{\omega_{\Lambda^{c}}}(\mathbf{x}, \mathbf{y}) \mid \omega_{\Lambda^{c}}\right)+\mathbb{E}\left(\mathbf{I}_{\omega^{\prime} \Lambda^{c}}(\mathbf{x}, \mathbf{y})-\mathbf{I}_{\omega_{\Lambda^{c}}}(\mathbf{x}, \mathbf{y}) \mid \omega_{\Lambda^{c}}^{\prime}\right) \\
& \geqq \mathbb{E}\left(\mathbf{I}\left(\mathbf{x} \sim \mathbf{y} \text { w.r.t. } \omega_{\Lambda^{c}}^{\prime} \text { and } \mathbf{x} \nsucc \mathbf{y} \text { w.r.t. } \omega_{\Lambda^{c}}\right) \mid \omega_{\Lambda^{\prime}}^{\prime}\right) .
\end{aligned}
$$

When the two configurations are not equivalent then, due to the strict positivity of the measure, the last term is strictly positive.

In this paper the Rising Tide Lemma was applied in Sect. 6. We expect it to be of interest also in various other situations where the FKG structure is of relevance.

Acknowledgements. It is a pleasure to thank I. Affleck and E.H. Lieb for stimulating discussions on topics related to this work, and C.M. Newman and A. Gandolfi for providing us with the reference [22].

\section{References}

1. Aizenman, M., Chayes, J.T., Chayes, L., Newman, C.M.: Discontinuity of the Magnetization in One-Dimensional $1 /|x-y|^{2}$ Ising and Potts Models, J. Stat. Phys. 50, 1-40 (1988)

2. Aizenman, M., Klein, A., Newman, C.: Percolation methods for disordered quantum Ising models. Preprint

3. Aizenman, M., Lieb, E.H.: Magnetic Properties of Some Itinerant-Electron Systems at $T>0$. Phys. Rev. Lett. 65, 1470-1473 (1990)

4. Aizenman, M., Martin, Ph.A.: Structure of Gibbs States of One-Dimensional Coulomb Systems. Commun. Math. Phys. 78, 99-116 (1980)

5. Aizenman, M., Nachtergaele, B.: Geometric aspects of quantum spin systems II. In preparation 
6. Affleck, I.: Exact results on the dimerization transition in $\mathrm{SU}(n)$ antiferromagnetic chains. J. Phys. C: Condens. Matter 2, 405-415 (1990)

7. Affleck, I., Lieb, E.H.: A proof of part of Haldane's conjecture on quantum spin chains. Lett. Math. Phys. 12, 57-69 (1986)

8. Baxter, R.J.: Exactly Solvable Models in Statistical Mechanics. London: Academic Press, 1982

9. Baxter, R.J.: Magnetisation discontinuity of the two-dimensional Potts model. J. Phys. A. Math. Gen. 15, 3329-3340 (1982)

10. Barber, M.N., Batchelor, M.T.: Spectrum of the biquadratic spin-1 antiferromagnetic chain. Phys. Rev. B40, 4621-4626 (1989)

11. Batchelor, M.T., Barber, M.: Spin-s quantum chains and Temperley-Lieb algebras. J. Phys. A: Math. Gen. 23, L15-L21 (1990)

12. Burton, R.M., Keane, M.: Density and uniqueness in percolation. Commun. Math. Phys. 121, 501-505 (1989)

13. Coniglio, A., Nappi, C.R., Peruggi, F., Russo, L.: Percolation and Phase Transitions in the Ising Model. Commun. Math. Phys. 51, 315-323 (1976)

14. Conlon, J.G., Solovej, J-Ph.: Random Walk Representations of the Heisenberg Model. J. Stat. Phys. 64, 251-270 (1991)

15. Cross, M.C., Fisher, D.S.: A new theory of the spin-Peierls transition with special relevance to the experiments on TTFCuBDT. Phys. Rev. B19, 402-419 (1979)

16. Dyson, F.J., Lieb, E.H., Simon, B.: Phase transitions in quantum spin systems with isotropic and non-isotropic interactions. J. Stat. Phys 18, 335-383 (1978)

17. Fannes, M., Nachtergaele, B., Werner, R.F.: Finitely correlated states for quantum spin chains. Commun. Math. Phys. 144, 443-490 (1992)

18. Fortuin, C.M.: On the random cluster model II and III. Physica 58, 393-418 (1972); Physica 59, 545-570 (1972)

19. Fortuin, C.M., Kasteleyn, P.W.: On the random cluster model I. Physica 57, 536-564 (1972)

20. Fortuin, C.M., Kasteleyn, P.W., Ginibre, L.: Correlation inequalities on some partially ordered sets. Commun. Math Phys. 22, 89-103 (1971)

21. Furstenberg, H.: Recurrence in Ergodic Theory and Combinatorial Number Theory. Princeton: Princeton University Press, 1981

22. Gandolfi, A., Keane, M., Russo, L.: On the uniqueness of the infinite occupied cluster in dependent two-dimensional site percolation. Ann. Prob. 16, 1147-1157 (1988)

23. Georgii, H.-O.: Gibbs measures and phase transitions. Berlin, New York: W. de Gruytter, 1988

24. Ginibre, J.: Reduced density matrices for the anisotropic Heisenberg model. Commun. Math. Phys. 10, 140-154 (1968)

25. Girvin, S.M., Arovas, D.P.: Hidden topological order in integer quantum spin chains. Physica Scripta T27, 156-159 (1989)

26. Goderis, D., Maes, C.: Constructing quantum dissipations and their reversible states from classical interacting spin systems. Ann. Inst. H. Poincaré 55, 805-828 (1991)

27. Hagiwara, M., Katsumata, K.: Observation of $S=\frac{1}{2}$ degrees of freedom in an undoped $S=1$ linear chain Heisenberg antiferromagnet. J. Phys. Soc. Japan. 61, 1481-1484 (1992)

28. Hagiwara, M., Katsumata, K., Affleck, I., Halperin, B.I., Renard, J.P.: Hyperfine structure due to the $S=\frac{1}{2}$ degrees of freedom in an $S=1$ linear chain antiferromagnet. J. Mag. Mag. Materials 104-107, 839-840 (1992)

29. Hagiwara, M., Katsumata, K., Renard, J.P., Affleck, I., Halperin, B.I.: Observation of $S=\frac{1}{2}$ degrees of freedom in an $S=1$ linear chain Heisenberg antiferromagnet. Phys. Rev. Lett. 65, 3181-3184 (1990)

30. Haldane, F.D.M.: Continuum dynamics of the 1-D Heisenberg antiferromagnet: Identification with the $\mathrm{O}(3)$ nonlinear sigma model. Phys. Lett. 93A, 464-468 (1983)

31. Hintermann, A., Kunz, H., Wu, F.Y.: Exact Results for the Potts Model in Two Dimensions. J. Stat. Phys. 19, 623-632 (1978)

32. Holley, R.: Remarks on the FKG Inequalities. Commun. Math. Phys. 36, 277-231 (1974)

33. Kennedy, T.: Long range order in the anisotropic quantum ferromagnetic Heisenberg model. Commun. Math. Phys. 100, 447-462 (1985)

34. Kennedy, T., Lieb, E.H.: Proof of the Peierls Instability in One Dimension. Phys. Rev. Lett. 59, 1309-1312 (1987)

35. Kennedy, T., Lieb, E.H., Shastri, B.S.: Existence of Néel order in some spin $1 / 2$ Heisenberg antiferromagnets. J. Stat. Phys. 53, 1019-1030 (1988) 
36. Kennedy, T., Tasaki, H.: Hidden Symmetry Breaking and the Haldane Phase in $S=1$ Quantum Spin Chains. Commun. Math. Phys. 147, 431-484 (1992)

37. Kirkwood, J.R., Thomas, L.E.: Expansions and Phase Transitions for the Ground State of Quantum Ising Lattice Systems. Commun. Math. Phys. 88, 569-580 (1983)

38. Klümper, A.: New Results for $q$-state Vertex Models and the Pure Biquadratic Spin-1 Hamiltonian. Europhys. Lett. 9, 815-820 (1989)

39. Klümper, A.: The spectra of $q$-state vertex models and related antiferromagnetic quantum spin chains. J. Phys. A: Math. Gen. 23, 809-823 (1990)

40. Kotecký, R., Laanait, L., Messager, A., Ruiz, J.: The $q$-state Potts Model in the Standard Pirogov-Sinai Theory: Surface Tensions and Wilson Loops. J. Stat. Phys. 58, 199-248 (1990)

41. Kotecký, R., Shlosman, S.B.: First-Order Phase Transitions in Large Entropy Lattice Models. Commun. Math. Phys. 83, 493-515 (1982)

42. Lieb, E.H., Mattis, D.C.: Ordering Energy Levels of Interacting Spin Systems. J. Math. Phys. 3, 749-751 (1962)

43. Lieb, E., Schulz, T., Mattis, D.: Two soluble models of an antiferromagnetic chain. Ann. Phys. (NY) 16, 407-466 (1961)

44. Matsui, T.: Uniqueness of the Translationally Invariant Ground State in Quantum Spin Systems. Commun. Math. Phys. 126, 453-467 (1990)

45. Matsui, T.: On Ground State Degeneracy of $\mathbb{Z}_{2}$-Symmetric Quantum Spin Models. Pub. RIMS (Kyoto) 27, 657-679 (1991)

46. Newman, C.M.: A general central limit theorem for FKG systems. Commun. Math. Phys. 91, 75-80 (1983)

47. den Nijs, M., Rommelse, K.: Preroughening transitions in crystal surfaces and valence bond phases in quantum spin systems. Phys. Rev. B40, 4709-4734 (1989)

48. Pitaevskii, L., Stringari, S.: Uncertainty principle, quantum fluctuations and broken symmetries. J. Low Temp. Phys 85, 377-388 (1991)

49. Shastri, B.S.: Bounds for correlation functions of the Heisenberg antiferromagnet. J. Phys. A: Math. Gen. 25, L249-253 (1992)

50. Sutherland, B.: Model for a multicomponent quantum system. Phys. Rev. B12, 3795-3805 (1975)

51. Sütö, A.: Percolation transition in the Bose gas. To appear in J. Phys. A: Math. Gen.

52. Thomas, L.E.: Quantum Heisenberg ferromagnets and stochastic exclusion processes. J. Math. Phys. 21, 1921-1924 (1980)

53. Tóth, B.: Improved lower bound on the thermodynamic pressure of the spin $1 / 2$ Heisenberg ferromagnet. Lett. Math. Phys. 28, 75-84 (1993)

54. Wu, F.Y.: The Potts model. Rev. Mod. Phys. 54, 235-268 (1982)

55. Affleck I.: The large- $N$ limit of quantum spin chains. Phys. Rev. Lett. 54, 966-969 (1985) [Reference added in proof]

Communicated by D.C. Brydges 
